\title{
1 Secreted microbial metabolites modulate gut immunity and inflammatory tone
}

2 Rabina Giri ${ }^{1,2}$, Emily C. Hoedt ${ }^{2,3}$, Khushi Shamsunnahar ${ }^{4}$, Michael A. McGuckin ${ }^{1,2}$, Mark

3 Morrison $^{2,3}$, Robert J. Capon ${ }^{4}$, Jakob Begun ${ }^{1,2 \#}$ and Páraic Ó Cuív ${ }^{2,3 \#}$

$4{ }^{1}$ Mater Research Institute - The University of Queensland, Translational Research Institute,

5 Brisbane, QLD, Australia

$6 \quad{ }^{2}$ Faculty of Medicine, The University of Queensland, St. Lucia, QLD, Australia

$7{ }^{3}$ The University of Queensland Diamantina Institute, The University of Queensland,

8 Translational Research Institute, Brisbane, QLD, Australia

$9{ }^{4}$ The Institute for Molecular Bioscience, The University of Queensland, Brisbane, QLD,

10 Australia

11 \#Equal contribution

12 Corresponding authors: Jakob Begun (Immunology, jakob.begun @ mater.uq.edu.au) \& Páraic

13 Ó Cuív (Microbiology, paraic.ocuiv@gmail.com)

14 Keywords: Crohn's disease, Ulcerative colitis, Anti-inflammatory, NF- $\kappa$ B, Gut microbiota,

15 Bioactive

16 Running title: Production of NF- $\mathrm{KB}$ suppressive bioactives by the human gut microbiota

17 Current addresses: ECH, APC Microbiome Institute \& Department of Microbiology, National

18 University of Ireland, Cork, Ireland; MMcG, The University of Melbourne, Victoria,

19 Australia; PÓC, Microba Life Sciences Ltd, Queensland, Australia. 


\section{Abstract}

21 Evidence is emerging that microbiome-immune system crosstalk regulates the tenor of host

22 intestinal immunity and predisposition to inflammatory bowel disease (IBD). We identified

23 five NF- $\mathrm{kB}$ suppressive strains affiliated with Clostridium clusters IV, XIVa and XV that

24 independently suppressed secretion of the chemokine IL-8 by peripheral blood mononuclear

25 cells and gut epithelial organoids from healthy human subjects, as well as patients with the

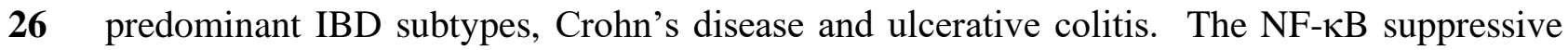

27 Clostridium bolteae AHG0001, but not C. bolteae BAA-613, suppressed cytokine-driven

28 inflammatory responses and endoplasmic reticulum stress in gut epithelial organoids derived

29 from Winnie mice that develop spontaneous colitis. This predicted in vivo responses thereby

30 validating a precision medicine approach to treat Winnie colitis and suggesting the microbiome

31 may function as an extrinsic regulator of host immunity. Finally, we identified a novel

32 molecule associated with NF- $\kappa \mathrm{B}$ suppression indicating gut bacteria could be harnessed to

33 develop new therapeutics.

\section{Introduction}

35 The human gut is the largest immune organ of the body and gut epithelial cells play a key role

36 in the establishment and maintenance of gut homeostasis, as well as rapid responses to

37 infection ${ }^{1}$. The gut is colonised by a diverse microbiota that has co-evolved with its host and

38 forms a durable symbiotic relationship through its modulation of innate and adaptive immune

39 responses ${ }^{2,3}$. However, with a few notable exceptions ${ }^{4,5}$ the microbes and microbial

40 determinants of immune tone remain cryptic.

41 Inflammatory bowel disease (IBD) is comprised of two predominant subtypes, termed Crohn's

42 disease (CD) and ulcerative colitis (UC), that are characterised by relapsing and remitting gut 
43 inflammation. The Nuclear factor $-\kappa \mathrm{B}(\mathrm{NF}-\kappa \mathrm{B})$ family of transcription factors are master

44 regulators of gut epithelial integrity and inflammation, activation of antigen presenting cells

45 and effector leukocytes, and are important contributors to the pathogenesis of IBD. Upon

46 activation, $\mathrm{NF}-\mathrm{\kappa B}$ dimers translocate to the nucleus where they regulate transcription of a wide

47 range of genes including those involved in immune and inflammatory responses ${ }^{6}$. In the

48 healthy gut, NF- $\kappa \mathrm{B}$ activation is tightly regulated ${ }^{7}$ however several IBD genetic risk alleles

49 including nod2, TOLLIP and A20 exert their pathogenic effects at least in part through

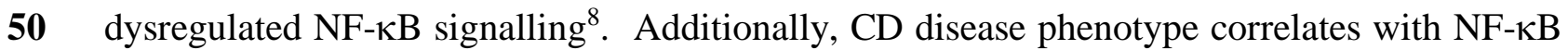

51 activation ${ }^{9}$, and macrophages and epithelial cells isolated from inflamed intestine of CD and

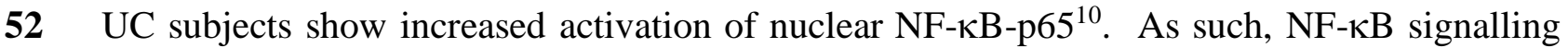

contributes significantly to the cascade of host-responses underlying the pathogenesis of IBD.

54 The gut microbiota is increasingly recognised as an important contributory risk factor for IBD.

55 Underlying this, the healthy and IBD gut microbiota differ and are characterised by structure-

56 function alterations to the microbiota ${ }^{11}{ }^{12}$, and faecal transplantation has proven effective in

57 some patients with $\mathrm{UC}^{13,14}$. Such findings suggest that key members of the microbiota regulate host inflammatory responses. Indeed, several bacterial taxa are not only more abundant in the healthy gut but can also suppress inflammatory responses and alleviate inflammation in animal models of disease ${ }^{15-17}$. These "anti-inflammatory" properties are best characterised for the gut

61 bacterium Faecalibacterium prausnitzii A2-165 which produces secreted peptides derived

62 from the Mam protein that suppress NF- $\kappa \mathrm{B}$ in human gut epithelial cells and murine colitis ${ }^{18}$.

63 However, while Firmicutes-affiliated Clostridia are amongst the most abundant and

64 functionally diverse gut bacteria, Mam is largely restricted to members of Faecalibacterium

65 spp., and much remains to be discovered about the immunomodulatory capacities inherent to other Firmicutes. 
67 Here, we identified five new Firmicutes isolates that are comparable to $F$. prausnitzii A2-165

68 in their NF-אB suppressive potency, and whose activities are characterised by strain specific

69 differences. Notably, these bacteria suppressed cytokine mediated IL-8 secretion in CD and

70 UC derived organoid cultures and peripheral blood mononuclear cells (PBMCs). Based on

71 these observations, we demonstrated using two Clostridium bolteae strains how a "precision

72 medicine" approach can be used to predict immunomodulatory and mucosal healing bioactivity

73 in vivo using the Winnie murine model of spontaneous colitis, demonstrating the potential of

74 bioprospecting the human microbiome for therapeutic leads.

\section{Results}

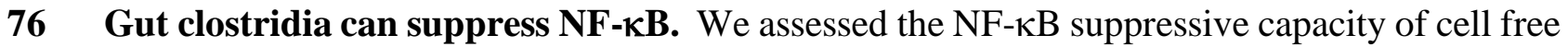
supernatants (CS) derived from 23 Firmicutes affiliated gut bacteria previously isolated by us via metaparental mating from a healthy pre-adolescent child ${ }^{19}$. The isolates were principally affiliated with Clostridium cluster XIVa, with several isolates also affiliated with clusters IV,

$80 \mathrm{XV}$ and XVIII. The isolates are distantly related to $F$. prausnitzii $\mathrm{A} 2-165$ and another NF-kB suppressive bacterium, Enterococcus faecalis AHG0090, that was also isolated by

82 metaparental mating ${ }^{20}$ (Figure 1A).

83 We assessed the ability of individual isolates to suppress NF- $\mathrm{BB}$ activation using LS174T 84 goblet cell-like and Caco-2 enterocyte like reporter cell lines ${ }^{21}$. The LS174T and Caco-2 cells 85 carry an NF- $\kappa \mathrm{B}$ inducible luciferase reporter gene and are responsive to TNF $\alpha$ and IL-1 $\beta$ 86 stimulation, respectively ${ }^{20,21}$. Although short chain fatty acids are posited to supress gut 87 inflammation, similar to previous reports ${ }^{22}$, the addition of up to $16 \mathrm{mM}$ of the short chain fatty acids acetate, butyrate and propionate did not activate NF- $\kappa \mathrm{B}$ under basal conditions.

89 However, all three short chain fatty acids enhanced cytokine-driven NF- $\kappa \mathrm{B}$ activation in a 
90 largely dose dependent manner (Supplementary Figure 1A-F). CS prepared from isolates

91 following growth in Modified Clostridial Medium (MCM) or Brain Heart Infusion (BHI)

92 medium were assessed for their ability to suppress NF- $\kappa$ B (Figure 1B). As previously

93 observed ${ }^{20,21}$, there was a high degree of concordance between the LS174T and Caco-2 reporter

94 cell lines with 7 strains identified that exhibited potent activities similar to $F$. prausnitzii A2-

95165 (Figure 1C, Z score $\leq-3$ ). In addition to F. prausnitzii A2-165, the isolates C. bolteae

96 AHG0001, Clostridium citroniae AHG0002 Pseudoflavonifractor sp. AHG0008, Clostridium

97 aldenense AHG0011, Eubacterium limosum AHG0013 and E. limosum AHG0017 suppressed

$98 \mathrm{NF}-\kappa \mathrm{B}$ in both cell lines when grown in MCM and/or BHI medium (Figure $1 \mathrm{C}, \mathrm{Z}$ score $\leq-3$ ).

99 The first pass screen was confirmed with CS prepared from individual isolates following

100 growth in MCM or BHI suppressing NF- $\kappa \mathrm{B}$ activation in both cell lines (Figure 1D-E,

$101 p<0.0001$ ). As anticipated, the NF-kB inhibitor indole-3-carbinol (I3C) and F. prausnitzii A2-

102165 suppressed cytokine-driven activation of the luciferase reporter in the LS174T and Caco-

1032 cell lines (Figure 1D-E, $p<0.0001$ ). Consistent with the reporter assay results, all the isolates

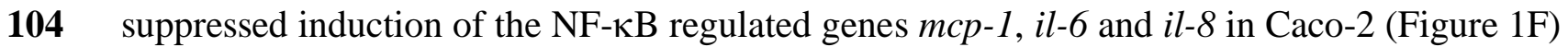

105 and LS174T (Supplementary Figure 1G) cells following stimulation. Critically, none of the

106 CS exhibited cytotoxic effects (Supplementary Figure 1H-I).

107 We also examined the NF-אB suppressive activity of CS prepared from the widely used

108 probiotic strains Lactobacillus rhamnosus GG (LGG), Lactobacillus casei Shirota (LCS),

109 Escherichia coli Nissle 1917 (Nissle) and Bifidobacterium animalis subsp. lactis BB-12

110 (Bifido). Notably, none of these strains suppressed cytokine driven NF- $\kappa B$ activation (Figure

111 (G) potentially explaining the limited efficacy of probiotics for the treatment of IBD ${ }^{23-27}$.

112 NF- $\mathrm{kB}$ suppression is strain specific. Having confirmed their suppressive activity, we next 
113 examined the intraspecies variations in NF-kB suppressive capacity. Isolates $C$. bolteae

114 AHG0001 and ATCC BAA-613 (OTU1), C. citroniae AHG0002 and AHG0004 (OTU2), and

115 C. aldenense AHG0011 and AHG0005 (OTU3) are assigned to the same operational taxonomic

116 units (Figure 1A, $\geq 97 \%$ 16S rRNA sequence identity). However, these OTUs were

117 characterised by marked intraspecies differences in their NF-אB suppressive capacities (Figure

118 2A-C). Next, we examined the apparent effect of growth medium on the suppressive effects

119 of $C$. bolteae AHG0001 and C. citroniae AHG0002 in the first pass screen. We determined

120 that CS prepared from C. bolteae AHG0001 and C. citroniae AHG0002 following growth in

121 MCM but not BHI suppressed TNF $\alpha$-driven mediated NF- $\kappa B$ activation in LS174T cells

122 (Figure 2D). Conversely, CS prepared from these strains following growth in BHI but not

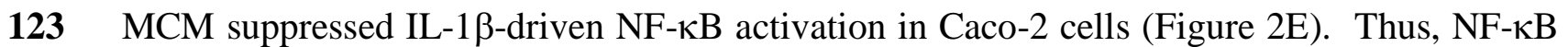

124 suppressive functionality is strain specific and nutritional influences on bioactive production

125 may affect the production of suppressive activity in vitro and the extent of anti-inflammatory

126 activity in situ in the gut.

127 We examined our collection of suppressive CS by a combination of size fractionation,

128 proteinase $\mathrm{K}$ and heat treatments to determine their biochemical characteristics. Using this

129 approach, we determined that the $\mathrm{NF}-\kappa \mathrm{B}$ suppressive activity for all strains except

130 Pseudoflavonifractor $\mathrm{sp}$. AHG0008 was predominantly associated with the $<3 \mathrm{kDa}$ fraction

131 (Figure 2F-K, Supplementary Results). Gut bacteria produce a structurally diverse array of

132 low molecular weight $\mathrm{NF}-\kappa \mathrm{B}$ suppressive bioactives ${ }^{18,20,28}$ and we focused on the $<3 \mathrm{kDa}$

133 fraction as we believed these bioactives would be more amenable to drug development. We

134 concluded that these bioactives could be broadly separated into two classes based on heat and

135 protease sensitivity (e.g. F. prausnitzii A2-165, C. aldenense AHG0011) or resilience (e.g. $C$.

136 bolteae AHG0001, C. citroniae AHG0002, and E. limosum AHG0017), possibly inclusive of 
137 both peptides and/or thermal and hydrolytically stable small molecules, respectively (Figures

138 2F-J, Supplementary Results). We sequenced the strains producing $<3 \mathrm{kDa}$ bioactives to near

139 completeness to identify candidate bioactive encoding biosynthetic gene clusters (BGCs)

140 (Table 1). Phylogenetic analysis using the Genome Taxonomy Database (GTDB) confirmed

141 the 16S rRNA based assignments (Supplementary Figure 2A). We also determined that the

142 strains exhibited a high degree of genome synteny with their near relatives (Supplementary

143 Figure 2B-E) and carried multiple BGCs (Table 1). None of the isolates encoded $F$. prausnitzii

144 Mam-like orthologs which is consistent with its narrow phylogenetic distribution ${ }^{18,29}$.

145 CS suppress ex vivo IL-8 secretion. IBD is challenging to treat due to the variability of

146 response to available medications. A proportion of this variability is related to underlying

147 genetic susceptibilities which likely drive their evolved immunophenotype and host-microbiota

148 relationship ${ }^{30,31}$. To assess whether the suppressive CS could affect epithelial inflammatory

149 responses in primary cells in the context of IBD associated genetic risk factors we assessed

150 their ability to prevent IL- $1 \beta$ driven IL-8 production in healthy $(n=6), C D(n=5)$ and UC $(n=5)$

151 derived primary intestinal epithelial organoid cultures. Interestingly, despite removal from the

152 inflammatory environment, there was significantly higher basal IL-8 production by organoids

153 derived from CD patients compared to those from non-IBD controls and UC patients (Figure

$1543 \mathrm{~A})$. Following stimulation with IL-1 $\beta$ there was a significantly more IL- 8 produced by organoids derived from UC but not CD when compared to healthy subjects (Figure 3B). As expected, IL-8 secretion was significantly inhibited by I3C, but not MCM in healthy, CD and

157 UC subjects. Treatment with $F$. prausnitzii A2-165 CS resulted in significantly suppressed IL-

1588 secretion when compared to the MCM control in healthy and CD but not UC subjects (Figure

159 3C-E). Treatment with CS from C. aldenense AHG0011, C. citroniae AHG0002, E. limosum

160 AHG0017, C. bolteae AHG0001 and Pseudoflavonifractor sp. AHG0008 significantly 
161 suppressed IL-8 secretion in healthy, CD and UC subjects, to an equivalent or greater degree

162 than $F$. prausnitzii A2-165 (Figure 3C-E). There was a high degree of concordance in the

163 degree of suppression between subjects within bacterial CS, in all subject groups, although

164 some subject specific differences were noted (Supplementary Figure 3A). Critically, we did

165 not observe any significant cytotoxic effects from the CS treatments (Supplementary Figure

166 3B-D).

167 In addition to effects on the epithelium, bioactives produced by gut bacteria may also be

168 absorbed and have systemic effects on immune cells. Therefore, the suppressive effects of the

169 CS on primary immune cells was examined using PBMCs collected from healthy, CD and UC

170 ( $n=6$ per group) subjects. While the basal concentrations of IL-8 released by PBMC from all

171 three groups were not significantly different (Figure 3F), their stimulation with TNF $\alpha$ resulted

172 in more IL-8 released from the PBMCs of the CD group in comparison to those prepared from

173 the healthy or UC groups (Figure 3G). As expected, IL-8 secretion by PBMCs from healthy,

$174 \mathrm{CD}$ and UC subjects was significantly inhibited by I3C and $F$. prausnitzii A2-165 CS (Figure

$1753 \mathrm{H}-\mathrm{J})$. Similarly, IL-8 secretion by PBMCs from healthy, CD and UC subjects was suppressed

176 by treatment with CS from $C$. aldenense AHG0011, C. citroniae AHG0002, E. limosum

177 AHG0017, C. bolteae AHG0001 and Pseudoflavonifractor sp. AHG0008; at least as

178 effectively as $F$. prausnitzii A2-165 (Figure 3H-J). There was limited variation in the response

179 to CS within the healthy, CD and UC subject groups although there were some subjects that

180 showed varying levels of suppression with individual CS (Supplementary Figure 4A).

181 Critically, we also did not observe any significant cytotoxic effects from the CS treatments on

182 PBMCs (Supplementary Figure 4B-D). Collectively, these results show that the CS of these

183 strains can suppress cytokine mediated inflammatory responses in the gut and immune

184 compartments in both an IBD and non-IBD genetic background. 
185 Precision treatment of murine colitis. The NF- $\kappa B$ pathway is highly conserved in mammals

186 and we next examined the ability of the CS to suppress IL-1 $\beta$ induced expression of the NF-

$187 \mathrm{\kappa B}$ regulated genes $\mathrm{Mip}-2$ and $\mathrm{Cxcl}-10$ in $\mathrm{C} 57 / \mathrm{B} 16$ derived murine organoids. All the CS tested

188 suppressed induction, suggesting that the bioactives likely act through conserved mammalian

189 cell targets (Figure 4A-B). We also examined the ability of the CS to suppress expression of

190 Mip-2 and Cxcl-10 in organoids derived from Winnie mice. Winnie mice carry a missense

191 mutation in $M u c 2$ that results in protein misfolding, endoplasmic reticulum (ER) stress and

192 defects in gut barrier function. These mice develop a spontaneous colitis characteristic of UC

193 and are an excellent preclinical model for human treatments ${ }^{32-34}$. We found that the majority

194 of CS significantly suppressed IL-1 $\beta$ induced expression of Mip-2 and Cxcl-10 on Winnie

195 derived organoids. However, in contrast to the findings in wild-type organoids, CS from $C$.

196 aldenense AHG0011 and F. prausnitzii A2-165 did not suppress Mip-2 and Cxcl-10 (Figure

197 4C-D). Furthermore, using Winnie derived gut epithelial organoids we determined C. bolteae

198 AHG0001 but not C. bolteae ATCC BAA-613 CS suppressed induction of Mip-2 and Cxcl-10

199 expression, confirming the strain specific differences observed in the reporter cell lines (Figure

200 4E). Interestingly, we also determined that $C$. bolteae AHG0001 but not $C$. bolteae ATCC

201 BAA-613 CS suppressed induction of the ER stress markers, Grp78 and $s X b p 1$, in Winnie

202 organoids (Figure 4E).

203 We hypothesised that functional capacity rather than phylogeny would be the principle

204 determinant of therapeutic efficacy and that primary organoid cultures could be used to predict

205 in vivo host responses to select $\mathrm{CS}$ in a precision medicine-based manner. To test this, CS

206 prepared from $C$. bolteae AHG0001 and ATCC BAA-613 were administered intrarectally for

20714 days to 6-week old Winnie mice with established colitis, as demonstrated by the elevated

208 diarrhoea scores at the start of the experiment (Figure 4F). C. bolteae AHG0001 CS 
significantly reduced diarrhoea scores over the course of the experiment compared to MCM and $C$. bolteae ATCC BAA-613 CS treated animals (Figure 4F). Furthermore, CS from $C$.

211 bolteae AHG0001 significantly reduced colonic inflammation as determined by a decreased

212 colon weight to length ratio (Figure 4G), histology scores (Figure 4H, Supplementary Figure

213 5A-C) and immune cell infiltration (Supplementary Figure 5E). Moreover, Winnie mice

214 treated with $C$. bolteae AHG0001 demonstrated increased mucin production and goblet cell

215 restitution in the distal and mid colon as determined by Alcian blue staining (Figure 4I,

216 Supplementary Figure 5D); indicative of reduced endoplasmic reticulum (ER) stress and

217 histologic healing. Consistent with reduced colitis, there was a significant reduction in colonic

218 expression of the inflammatory genes $\mathrm{Il}-6$ and $\mathrm{Cxcl}-10$ and the ER stress markers spliced-Xbpl

219 and Grp78 in the colon (Figure 4J). Together, these results showed the feasibility of applying

220 a precision medicine approach using ex vivo organoid cultures to accurately predict treatment

221 response in colitis.

222 Finally, we hypothesised that intraspecies variations in $\mathrm{NF}-\kappa \mathrm{B}$ suppressive capacity, together

223 with the influence of culture media on bioactive production, could facilitate identification of candidate bioactive encoding BGCs and/or bioactive molecules produced by $C$. bolteae using comparative genomics or metabolomics. Comparative genomic analyses revealed that $C$. bolteae AHG0001 carries 19 predicted BGCs of which 14 are either highly or partially conserved in C. bolteae ATCC BAA-613 (Figure 5A, Supplementary Figure 6A). However,

228 as the biosynthesis of bioactives by gut bacteria may be principally driven through modest

229 modifications of common primary metabolites ${ }^{35,36}$ we considered it likely that other BGCs

230 would be overlooked by in silico screens. We consequently applied a process of bioassay

231 guided solvent extractions and filtrations, followed by ultra-high-performance liquid

232 chromatography quadrupole time-of-flight mass spectrometric analysis (UPLC-QTOF), and 
233 comparative metabolomics, to identify the bioactive(s) (Figure 5B, Supplementary

234 Information). These analyses successfully identified a cluster of six structurally related small

235 molecules (Figure 5C, 5Ca, i-vi) that were uniquely present in the NF- $\kappa \mathrm{B}$ suppressive ethyl

236 acetate (EtOAc) extract of $C$. bolteae AHG0001, but were absent in comparable extracts of $C$.

237 bolteae ATCC BAA-613, and, following semi-preparative HPLC fractionation of the $C$.

238 bolteae AHG0001 EtOAc extract, were uniquely localised in the NF- $\kappa \mathrm{B}$ suppressive fractions

239 (Supplementary Figure 6B-C). The identification of a novel secreted molecule confirmed the

240 benefit of using an integrated approach combining bacterial isolation, functional screens and

241 comparative metabolomics to expedite bioactive discovery.

\section{Discussion}

243 Firmicutes affiliated bacteria are amongst the most abundant gut microbes and these taxa are

244 widely recognised to possess immunomodulatory capacities ${ }^{15,37,38}$. However, they are poorly

245 represented in culture collections and their ability to modulate immune responses remain

246 largely undefined. In this study, we identified five gut bacterial strains affiliated with

247 Clostridium clusters IV, XIVa and XV that are comparable to the well-characterised $F$.

248 prausnitzii A2-165 strain in their ability to suppress NF- $\kappa \mathrm{B}$ activation. The NF- $\kappa \mathrm{B}$ suppressive

249 bioactivities were characterised by significant biochemical and intraspecies variations

250 suggesting there may be extensive functional redundancy and NF- $\kappa \mathrm{B}$ suppressive capacity may

251 be more prevalent than previously appreciated. This is consistent with Geva-Zatorsky et al., ${ }^{2}$

252 who determined that as few as 53 isolates were associated with over 24,000 immune

253 phenotypes that include functionalities relevant to IBD (e.g. Treg induction). Modulating host

254 immune responses may support the ability of gut bacteria to colonise and persist in the gut environment and the ability of the microbiota to act as an extrinsic regulator of host immunity 
individuals.

258 IBD is characterised by a dysregulated immune response with select genetic susceptibilities

259 affecting therapeutic responsiveness ${ }^{30,31}$. In order to develop improved precision treatments

260 for IBD we therefore used gut epithelial organoids and immune cells to identify bacteria

261 capable of supressing cytokine mediated inflammatory responses. The heat and proteinase K

262 resilient bioactives showed strong suppression of IL-8 secretion in organoids and immune cells

263 from healthy, CD and UC subjects. Interestingly, the putative peptide bioactives produced by

$264 F$. prausnitzii A2-165 and $C$. aldenense AHG0011 were notably less suppressive in UC derived

265 organoids and PBMCs, and CD organoids, when compared to organoids derived from healthy

266 controls; this may be reflective of the increased endogenous protease activity in IBD ${ }^{39}$. Our in

267 vitro and ex vivo data also suggested that functional capacity rather than phylogeny may be the

268 key determinant of biologic effects. To explore this hypothesis, we capitalised on the $C$.

269 bolteae intraspecies differences and demonstrated that a precision medicine approach could be applied to alleviate established colitis in Winnie mice. Notably, treatment with $C$. bolteae

271 AHG0001 CS was associated with a rapid onset of action with improvement in diarrhoea,

272 alleviation of inflammation and ER stress, as well as restoration of goblet cell numbers and mucin production. Mucosal and histologic healing are amongst amongst the best predictors of

274 long-term outcomes in IBD and taken together our data suggests a precision medicine approach

275 could be applied to microbiome based IBD treatment.

276 The NF- $\mathrm{BB}$ suppressive strains carry multiple BGCs, many of whose products remain cryptic,

277 underlining the inherent challenges in applying genomic based approaches to map genotype

278 with phenotype. In addition, the biosynthesis of bioactives by gut bacteria may be principally

279 driven through modest modifications of common primary metabolites that are underpinned by

280 small BGCs ${ }^{35,40}$. As the medium dependent effects on NF- $\kappa \mathrm{B}$ suppression may affect the 
281 therapeutic efficacy of live biotherapeutics for IBD, we therefore used a bioassay-guided

282 fractionation and a comparative metabolomic approach to identify a novel low molecular

283 weight non-polar molecule that was associated with the NF-kB suppressive activity of $C$.

284 bolteae AHG0001. Critically, this molecule was not associated with medium components

285 suggesting the suppressive activity is unlikely to be due to the biotransformation of medium

286 components ${ }^{41}$. Consistent with other microbial bioactives, the C. bolteae AHG0001 bioactive

287 acts independently of the bacterial cell and suppresses the inflammatory response in animals.

288 In summary, our IBD guided approach provides new opportunities to rationally bioprospect

289 the gut microbiota for precision live biotherapeutic strains and/or bioactives that could be used

290 to expedite the development of safer and more efficacious therapeutics. 
292 Bacterial strains, culture conditions and analyses. Firmicutes affiliated bacteria were

293 cultured in anoxic MCM or BHI medium (Supplementary Information). E. coli Nissle 1917

294 was cultured using LB medium. L. casei Shirota was isolated from a Yakult Original probiotic

295 drink and cultured using anoxic de Man Rogosa Sharpe (MRS) or BHI medium. L. rhamnosus

296 GG and B. animalis subsp. lactis BB-12 were isolated from a probiotic capsule and cultured

297 using anoxic MRS or BHI medium.

298 Bacterial comparative analyses. The phylogeny of the Firmicutes isolates was inferred using

299 the $r$ rs gene sequences as described in the Supplementary Information. High molecular weight

300 DNA was prepared and sequenced using the Illumina NextSeq 500 system $(2 \times 150 \mathrm{bp}$ High

301 Output kit) with v2 chemistry as previously described ${ }^{20}$. The C. bolteae AHG0001, C.

302 citroniae AHG0002, C. aldenense AHG0011 and E. limosum AHG0017 sequence data were

303 assembled, assessed for contamination and completeness and ordered as described in the

304 Supplementary Information. Genome based phylogeny was determined using the Genome

305 Taxonomy Database (GTDB) as previously described ${ }^{20}$. Candidate BGC were identified and

306 characterised as described in the Supplementary Information. The Whole Genome Shotgun

307 projects for $C$. bolteae AHG0001, C. citroniae AHG0002, C. aldenense AHG0011 and E.

308 limosum AHG0017 were deposited respectively at DDBJ/EMBL/GenBank under the

309 accessions QYRW00000000, QYRX00000000, QYRY00000000 and QYRZ00000000. The

310 version described in this paper are the first versions, [XXXX]01000000.

311 Measurement of immunomodulatory activities. The immunomodulatory potential of the

312 individual strains was examined following growth in MCM, BHI or MRS. Briefly, for the first

313 pass screen an individual colony was used to inoculate medium and the culture was grown for 
314 up to 96 hours. For the confirmatory screens, the NF- $\kappa B$ suppressive capacity of biological

315 replicate cultures produced from select strains was assessed. Briefly, two independent broth

316 cultures were established from individual colonies of each strain $(n=2$ independent biological

317 replicates per strain) and following growth as described above, each individual culture was

318 used to inoculate 3 tubes of broth ( $n=6$, consisting of $n=2$ independent biological replicates per

319 strain with $n=3$ technical replicate for each biological replicate). The cultures were grown until

320 early stationary phase and then $1.5 \mathrm{ml}$ of each culture was centrifuged at $25,000 \mathrm{x}$ g for 3

321 minutes. Then, $1 \mathrm{ml}$ of the cell-free supernatant fraction was collected and stored at $\leq 30^{\circ} \mathrm{C}$ as

322 a single-use aliquot. The NF- $\mathrm{B}$ suppressive capacity of the $\mathrm{CS}$ was assessed using the

323 LS174T-NF-kBluc or Caco-2-NF-kBluc reporter cell assays adapted for high-throughput

324 screening (Supplementary Information). The effects of sodium salt SCFA on NF- $\mathrm{B}$ activation

325 were assessed by treating the cell lines for $30 \mathrm{~min}$ and then determining their ability to suppress

326 cytokine mediated NF- $\mathrm{BB}$ activation as described above. The cytotoxicity of the supernatants

327 was assessed using the CytoTox96® Non-Radioactive Cytotoxicity Assay according to the manufacturer's instructions (Promega).

Organoid culturing and immunomodulatory assays. All patient samples were collected in

331 Committee (HREC 2016001782 \& HREC/14/MHS/125) for the Mater Inflammatory Bowel

332 Disease Biobank. Colonic biopsies (6 x 3mm pinch biopsies) were collected from healthy

$333(n=6), C D(n=5)$ and UC ( $n=5)$ patients (Supplementary Table 1). The colonic biopsies were

334 processed and cultured as previously described (Supplementary Information). To assess the

335 ability of the CS to suppress IL-8 secretion the organoids were seeded in a 48 well plate and

336 grown for 48 hours. Then, organoids were treated with $10 \% \mathrm{v} / \mathrm{v}$ of select CS in $50 \% \mathrm{~L}-\mathrm{WRN}$

337 conditioned medium and subsequently stimulated with rhIL-1 $\beta\left(50 \mathrm{ng} \cdot \mathrm{ml}^{-1}\right)$ for 24 hours before 
338 quantifying IL-8 in the supernatant. Cytotoxicity was assessed using the CytoTox 96® Non-

339 Radioactive Cytotoxicity Assay. For the animal experiments, colonic tissues from C57BL/6

340 and Winnie $(\mathrm{n}=2)$ mice were segmented and the crypts were isolated and cultured according to

341 previously established protocols (Supplementary Information). For the treatments, the

342 organoids were first seeded in a 24 well plate and grown for 48 hours. The organoids were then

343 pre-treated with $10 \% \mathrm{v} / \mathrm{v}$ of select CS for $30 \mathrm{mins}$ and then stimulated with $50 \mathrm{ng} / \mathrm{ml} \mathrm{mIL-1 \beta}$

344 for 6 hours. The cells were lysed and used for mRNA expression.

345 Peripheral Blood Mononuclear Cell (PBMC) isolation and immunomodulatory assays.

346 Human peripheral blood was obtained for 6 healthy, CD and UC patients from the Mater

347 Inflammatory Bowel Disease biobank. PBMCs were isolated by Ficoll gradient density

348 centrifugation (Supplementary Information). For the treatments, 500,000 cells per well were

349 plated on a 96-well plate and treated with $10 \% \mathrm{v} / \mathrm{v}$ of CS in RPMI medium for 30 minutes,

350 followed by stimulation with $\operatorname{rhTNF} \alpha(50 \mathrm{ng} / \mathrm{ml})$. IL-8 secretion and cytotoxicity was assessed

351 as previously described.

352 RNA extraction, cDNA synthesis and gene expression. Total RNA was prepared as previously described except that LS174T and Caco-2 cells were used. The expression of il6, $i l 8$ and $c x c l-10$ was assessed as previously described ${ }^{20,21}$. RNAeasy mini kits (QIAGEN) were used to extract RNA from the mouse organoid cultures according to the manufacturer's

356 instructions. RNA was reverse transcribed to cDNA using iScript cDNA synthesis kit (Bio-

357 Rad Laboratories) and the protocol provided by the manufacturer. The $\mathrm{C}_{\mathrm{t}}$ values for each gene

358 were normalised to untreated controls and further normalised to housekeeping gene (mouse $\beta$ 359 actin) and presented as fold change. The mouse primers used are summarised in 360 Supplementary Table 2). 
361 Quantitative cytokine expression assays. To quantify IL-8 secretion, cell free supernatant was

362 collected after 24 hours and IL-8 was quantified by ELISA according to manufacturer's

363 instructions (BioLegend).

364 Animal experiments. All animal experiments were approved by the University of Queensland

365 Animal Ethics Committee. Winnie mice were bred in-house in a pathogen-free animal facility.

366 Male and female mice were intrarectally gavaged with $50 \mu \mathrm{l}$ of CS from C. bolteae AHG0001

367 and ATCC BAA-613 for 14 days. MCM medium processed in the same manner as the CS was

368 used as the vehicle control. Disease activity was assessed as described in the Supplementary

369 Information.

370 GNPS Analyses. UHPLC-QTOF (Agilent Technologies 6545 Q-TOF LC/MS) data was

371 acquired by subjecting aliquots of EtOAc extracts obtained from (a) cultures of $C$. bolteae

372 AHG0001 in either MCM or BHI media, (b) cultures of $C$. bolteae BAA-613, in either MCM

373 or BHI media, and; (c) un-inoculated MCM and BHI media $(1 \mu \mathrm{L})$. UHPLC conditions were

374 as described in the Supplementary Information. The acquired MS/MS data was converted from

375 Agilent MassHunter data file (.d) to the mzXML file format using the software MS-Convert ${ }^{42}$.

376 Molecular networks were generated using the online Global Natural Products Social molecular

377 networking web-platform (GNPS) (gnps.ucsd.edu). MS-Cluster with a precursor ion mass

378 tolerance of $0.02 \mathrm{Da}$ and a MS/MS fragment ion tolerance of $0.02 \mathrm{Da}$ were selected to create

379 consensus spectra ${ }^{43}$. A minimum cluster size of 1 , cosine score 0.7 , and minimum number of

380 fragments of 6 , were selected for molecular networking. The spectral networks were imported

381 into Cytoscape 3.5.1 ${ }^{44}$ and visualized using force directed layout where nodes represented

382 parent masses.

383 Analytical fractionation of $\mathrm{NF}$ - $\boldsymbol{k B}$ suppressive extract. An EtOAc extract (3mg) of C. bolteae 
384 AHG0001 cultivated in MCM medium was subjected to analytical HPLC (Supplementary

385 Information) to yield 17 fractions. Each fraction was dried in vacuo then resuspended in $\mathrm{MeOH}$

$386(50 \mu \mathrm{L})$. NF- $\kappa \mathrm{B}$ suppressive fractions were combined and an aliquot $(1 \mu \mathrm{L})$ was subjected to

387 UHPLC-QTOF analysis, with single ion extraction (SIE) same quadrupole time-of-flight

388 spectrometer and UHPLC conditions described above. Single ion extraction $(\mathrm{m} / \mathrm{z}$, molecular

389 ion) chromatograms for molecules exclusively present in C. bolteae AHG0001 (i.e. i-xvi in

390 GNPS analysis Figure 5C), using Agilent MassHunter Qualitative Analysis software,

391 confirmed that only i-vi were present in the NF- $\mathrm{BB}$ suppressive fraction.

392 Statistical analyses. The NF- $\kappa \mathrm{B}$ suppressive effects of the suppressive strains was assessed

393 using biological duplicates with each duplicate comprising of three technical replicates.

394 Significance was determined using a one-way ANOVA with correction for multiple

395 comparisons with a Dunnett test. Differences were considered significant at $p \leq 0.05$. A heat

396 map of the first pass screen data was produced using GraphPad Prism (version 7.0) and the

397 Heatmap tool at the HIV sequence database

398 (https://www.hiv.lanl.gov/content/sequence/HEATMAP/heatmap.html). The animal

399 experiments were performed twice independently, and the data combined for analysis. The

400 D'Agostino-Pearson omnibus test was used to verify the normal distribution of all data.

401 Significance was determined using t-tests, one-way ANOVA with multiple comparisons

402 (Sidak), two-way ANOVA corrected for multiple comparisons with a Dunnett test using

403 GraphPad Prism (version 7.0). Differences were considered significant at $p \leq 0.05$.

\section{Acknowledgements}

405 This research was supported via funds provided by the University of Queensland (UQ) Faculty

406 of Medicine (MM, JB and PÓC) and Diamantina Institute (MM). We gratefully acknowledge 
407 the support provided by the UQ Research Training Program and Mater Frank Clair Scholarship

408 (RG), UQ Institute for Molecular Bioscience (KS and RKC) and UQ Reginald Ferguson

409 Fellowship in Gastroenterology (PÓC). The Translational Research Institute is supported by a

410 grant from the Australian Government.

\section{Author Contributions}

412 PÓC and JB conceived the study and developed it with MMcG and MM; PÓC and RG prepared

413 samples for analysis and performed the immunomodulatory characterizations; RG and JB

414 performed the organoid, PBMC and animal experiments; ECH and PÓC performed the

415 genomic analyses; KS and RJC performed the metabolomics and molecule analyses; RG, ECH,

$416 \mathrm{KS}, \mathrm{MMcG}, \mathrm{MM}, \mathrm{RJC}$ JB and PÓC analysed the data, and; PÓC wrote the manuscript with

417 RG, ECH, KS, MMcG, MM, RJC and JB.

\section{Competing Interest}

419 The authors declare no competing interest. 


\section{References}

422

423

424

425

426

427

428

429

430

431

432

433

434

435

436

437

438

439

440

441

442

443

444

445

446

447

448

449

450

451

452

453

454

455

456

457

458

459

460

461

462

463

464

465

466

467

1. Peterson, L.W. \& Artis, D. Intestinal epithelial cells: regulators of barrier function and immune homeostasis. Nature Reviews Immunology 14, 141 (2014).

2. Geva-Zatorsky, N. et al. Mining the human gut microbiota for immunomodulatory organisms. Cell 168, 928-943.e911 (2017).

3. Kabat, A.M., Srinivasan, N. \& Maloy, K.J. Modulation of immune development and function by intestinal microbiota. Trends Immunol. 35, 507-517 (2014).

4. Mazmanian, S.K., Round, J.L. \& Kasper, D.L. A microbial symbiosis factor prevents intestinal inflammatory disease. Nature 453, 620-625 (2008).

5. Wlodarska, M. et al. Indoleacrylic acid produced by commensal Peptostreptococcus species suppresses inflammation. Cell Host Microbe 22, 25-37.e26 (2017).

6. Wullaert, A., Bonnet, M.C. \& Pasparakis, M. NF- $\mathrm{kB}$ in the regulation of epithelial homeostasis and inflammation. Cell Res. 21, 146-158 (2011).

7. Renner, F. \& Schmitz, M.L. Autoregulatory feedback loops terminating the NFkappaB response. Trends Biochem. Sci. 34, 128-135 (2009).

8. Zaidi, D. \& Wine, E. Regulation of nuclear factor kappa-light-chain-enhancer of activated B Cells $(\mathrm{NF}-\kappa \beta)$ in inflammatory bowel diseases. Frontiers in Pediatrics 6 (2018).

9. Han, Y.M. et al. NF-kappa B activation correlates with disease phenotype in Crohn's disease. PLoS One 12, e0182071 (2017).

10. Rogler, G. et al. Nuclear factor kappaB is activated in macrophages and epithelial cells of inflamed intestinal mucosa. Gastroenterology 115, 357-369 (1998).

11. Gevers, D. et al. The treatment-naive microbiome in new-onset Crohn's disease. Cell Host \& Microbe 15, 382-392 (2014).

12. Franzosa, E.A. et al. Gut microbiome structure and metabolic activity in inflammatory bowel disease. Nat Microbiol 4, 293-305 (2019).

13. Costello, S.P. et al. Systematic review with meta-analysis: faecal microbiota transplantation for the induction of remission for active ulcerative colitis. Aliment. Pharmacol. Ther. 46, 213-224 (2017).

14. Wilson, B.C., Vatanen, T., Cutfield, W.S. \& O'Sullivan, J.M. The super-donor phenomenon in fecal microbiota transplantation. Frontiers in cellular and infection microbiology 9, 2-2 (2019).

15. Sokol, H. et al. Faecalibacterium prausnitzii is an anti-inflammatory commensal bacterium identified by gut microbiota analysis of Crohn disease patients. Proc. Natl. Acad. Sci. U. S. A. 105, 16731-16736 (2008).

16. Eeckhaut, V. et al. Butyricicoccus pullicaecorum in inflammatory bowel disease. Gut 62, 1745-1752 (2012).

17. Takeshita, K. et al. A single species of Clostridium subcluster XIVa decreased in ulcerative colitis patients. Inflamm. Bowel Dis. 22, 2802-2810 (2016).

18. Quevrain, E. et al. Identification of an anti-inflammatory protein from Faecalibacterium prausnitzii, a commensal bacterium deficient in Crohn's disease. Gut 65, 415-425 (2016).

19. Ó Cuív, P. et al. Isolation of genetically tractable most-wanted bacteria by metaparental mating. Sci. Rep. 5, 13282 (2015).

20. Ó Cuív, P. et al. Enterococcus faecalis AHG0090 is a genetically tractable bacterium and produces a secreted peptidic bioactive that suppresses nuclear factor kappa B activation in human gut epithelial cells. Front. Immunol. 9 (2018). 
21. Ó Cuív, P. et al. The gut bacterium and pathobiont Bacteroides vulgatus activates NFkappaB in a human gut epithelial cell line in a strain and growth phase dependent manner. Anaerobe 47, 209-217 (2017).

22. Lakhdari, O. et al. Identification of NF- $\mathrm{BB}$ modulation capabilities within human intestinal commensal bacteria. Journal of Biomedicine and Biotechnology 2011 (2011).

23. Ghoshal, U.C. et al. The role of the microbiome and the use of probiotics in gastrointestinal disorders in adults in the Asia-Pacific region - background and recommendations of a regional consensus meeting. J. Gastroenterol. Hepatol. 33, 5769 (2018).

24. Bousvaros, A. et al. A randomized, double-blind trial of Lactobacillus GG versus placebo in addition to standard maintenance therapy for children with Crohn's disease. Inflamm. Bowel Dis. 11, 833-839 (2005).

25. Prantera, C., Scribano, M.L., Falasco, G., Andreoli, A. \& Luzi, C. Ineffectiveness of probiotics in preventing recurrence after curative resection for Crohn's disease: a randomised controlled trial with Lactobacillus GG. Gut 51, 405-409 (2002).

26. Petersen, A.M. et al. Ciprofloxacin and probiotic Escherichia coli Nissle add-on treatment in active ulcerative colitis: a double-blind randomized placebo controlled clinical trial. J Crohns Colitis 8, 1498-1505 (2014).

27. Wildt, S., Nordgaard, I., Hansen, U., Brockmann, E. \& Rumessen, J.J. A randomised double-blind placebo-controlled trial with Lactobacillus acidophilus La-5 and Bifidobacterium animalis subsp. lactis BB-12 for maintenance of remission in ulcerative colitis. J Crohns Colitis 5, 115-121 (2011).

28. Kaci, G. et al. Inhibition of the NF-kappaB pathway in human intestinal epithelial cells by commensal Streptococcus salivarius. Appl. Environ. Microbiol. 77, 46814684 (2011).

29. Martín, R. et al. Functional characterization of novel Faecalibacterium prausnitzii strains isolated from healthy volunteers: A step forward in the use of $F$. prausnitzii as a next-generation probiotic. Front. Microbiol. 8 (2017).

30. Niess, J.H. et al. NOD2 polymorphism predicts response to treatment in Crohn's disease - first steps to a personalized therapy. Dig. Dis. Sci. 57, 879-886 (2012).

31. Barber, G.E. et al. Genetic markers predict primary non-response and durable response to anti-TNF biologic therapies in Crohn's disease. Am. J. Gastroenterol. 111, 1816-1822 (2016).

32. Oancea, I. et al. Colonic microbiota can promote rapid local improvement of murine colitis by thioguanine independently of T lymphocytes and host metabolism. Gut 66, 59-69 (2017).

33. Das, I. et al. Glucocorticoids alleviate intestinal ER stress by enhancing protein folding and degradation of misfolded proteins. J. Exp. Med. 210, 1201-1216 (2013).

34. Wang, R. et al. Neutralizing IL-23 is superior to blocking IL-17 in suppressing intestinal inflammation in a spontaneous murine colitis model. Inflamm. Bowel Dis. 21, 973-984 (2015).

35. Cohen, L.J. et al. Commensal bacteria make GPCR ligands that mimic human signalling molecules. Nature 549, 48-53 (2017).

36. Colosimo, D.A. et al. Mapping interactions of microbial metabolites with human Gprotein-coupled receptors. Cell Host \& Microbe 26, 273-282.e277 (2019).

37. Atarashi, K. et al. Treg induction by a rationally selected mixture of Clostridia strains from the human microbiota. Nature 500, 232-236 (2013).

38. Atarashi, K. et al. Induction of colonic regulatory T cells by indigenous Clostridium 
species. Science 331, 337-341 (2011).

39. Vergnolle, N. Protease inhibition as new therapeutic strategy for GI diseases. Gut 65,

40. Cohen, L.J. et al. Functional metagenomic discovery of bacterial effectors in the human microbiome and isolation of commendamide, a GPCR G2A/132 agonist. Proc. Natl. Acad. Sci. U. S. A. 112, E4825-4834 (2015).

41. Theilmann, M.C. et al. Lactobacillus acidophilus metabolizes dietary plant glucosides

42. Kessner, D., Chambers, M., Burke, R., Agus, D. \& Mallick, P. ProteoWizard: open and externalizes their bioactive phytochemicals. MBio 8 (2017). source software for rapid proteomics tools development. Bioinformatics 24, 25342536 (2008).

43. Frank, A.M. et al. Clustering millions of tandem mass spectra. J. Proteome Res. 7, 113-122 (2008).

44. Shannon, P. et al. Cytoscape: a software environment for integrated models of biomolecular interaction networks. Genome Res. 13, 2498-2504 (2003). 
A

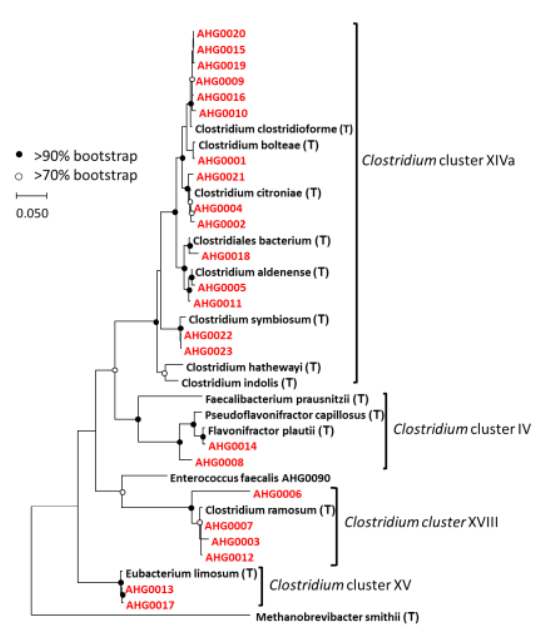

C

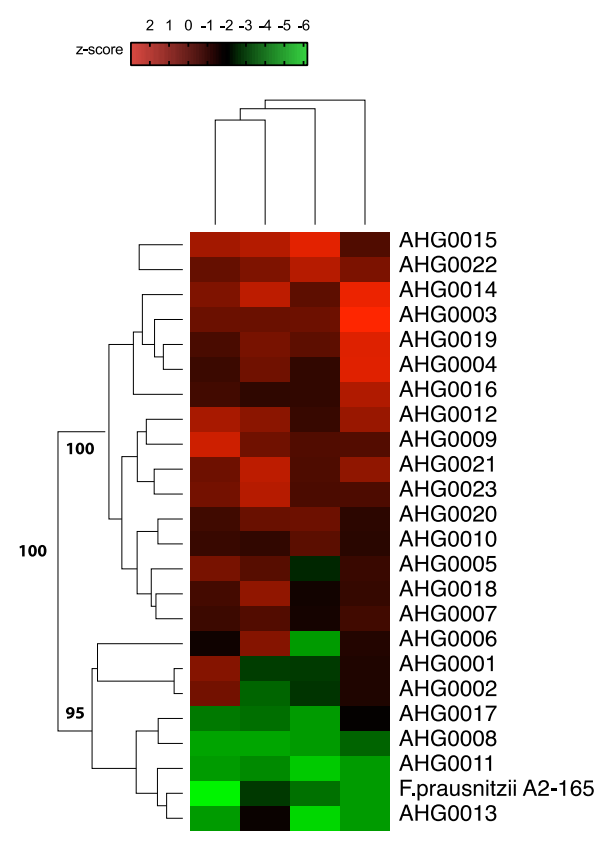

B

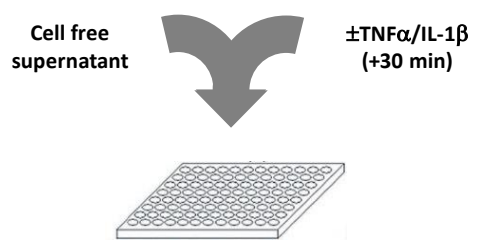

LS174T goblet cell like cell line Caco-2 enterocyte like cell line

Identify NF-KB suppressive isolates

D

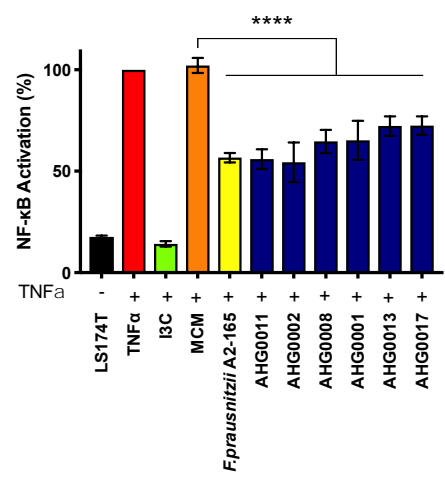

E

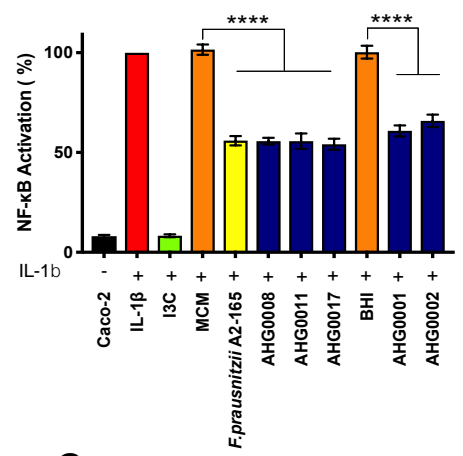

$\mathbf{F}$

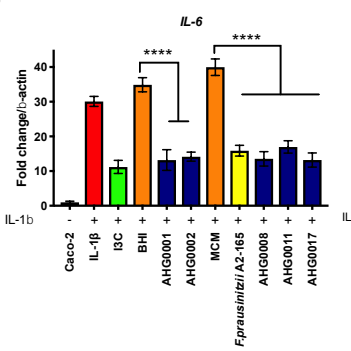

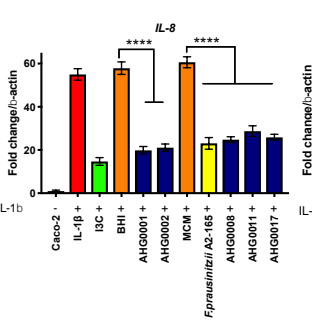

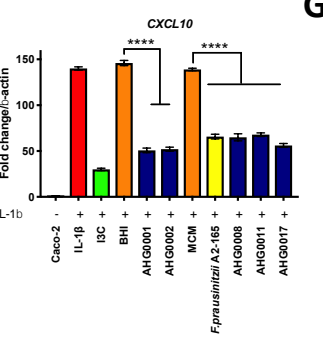

G

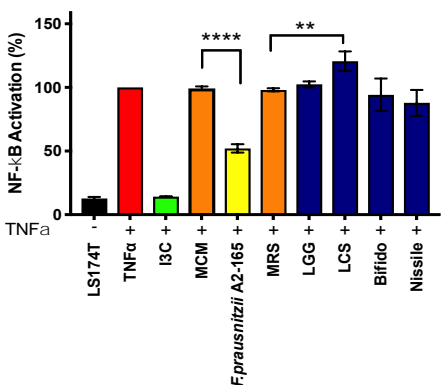

534 Figure 1A. 16S rRNA based phylogeny of the MPM isolates characterised in this study (red

535 typeface) and representative microbial isolates and reference sequences (bold black typeface). 
537 of the bacteria examined in this study. Cell free CS was added to the reporter cell lines which

538 were then stimulated with cytokine after $30 \mathrm{~min}$. Luciferase activity was assayed after 4 hours.

539 C. Heat map analysis of the NF-kB suppressive capacity of the bacterial isolates. The ability

540 of CS prepared from bacterial isolates grown in MCM or BHI to suppress NF- $\kappa \mathrm{B}$ in LS174T

541 or Caco-2 reporter cell lines was assessed twice independently. A Z-factor of $0.805 \pm 0.06$

$542(\mathrm{MCM})$ and $0.87 \pm 0.01(\mathrm{BHI})$ was achieved for the LS174T cells while a Z-factor of $0.78 \pm$

$5430.057(\mathrm{MCM})$ and $0.765 \pm 0.02(\mathrm{BHI})$ was achieved for the Caco-2 cells. A subset of strains

544 formed an NF- $\kappa \mathrm{B}$ suppressive cluster with $F$. prausnitzii A2-165. D. LS174T based

545 confirmatory assay of the hits identified from the first pass screen. NF- $\kappa \mathrm{B}$ activation was

546 assessed $4 \mathrm{~h}$ after $\mathrm{TNF} \alpha$ stimulation and the extent of suppression was assessed against sterile

547 medium (mean (standard deviation (SD))). E. Caco-2 based confirmatory assay of the hits

548 identified from the first pass screen. NF- $\kappa$ B activation was assessed $4 \mathrm{~h}$ after IL- $1 \beta$ stimulation

549 and the extent of suppression was assessed against sterile medium (mean (SD)). F. Caco-2

550 based qRT-PCR confirmatory assay of the hits identified from the first pass screen (mean

551 (SD)). F. prausnitzii A2-165 and the validated hits suppress IL-1 $\beta$ induced cxcl10, il6 and il8

552 expression in Caco-2 cells. G. Analysis of the ability of L. rhamnosus GG (LGG), L. casei

553 Shirota (LCS), E. coli Nissle 1917 (Nissle) and B. animalis subsp. lactis BB-12 (Bifido) to

554 suppress TNF $\alpha$ mediated activation of NF-kB in LS174T cells. Cell free CS prepared from

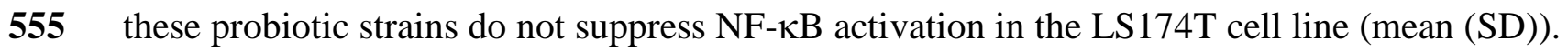

$556 * * p<0.01, * * * * p<0.0001$ as determined by one-way ANOVA with Dunnett's multiple

557 comparison test. 

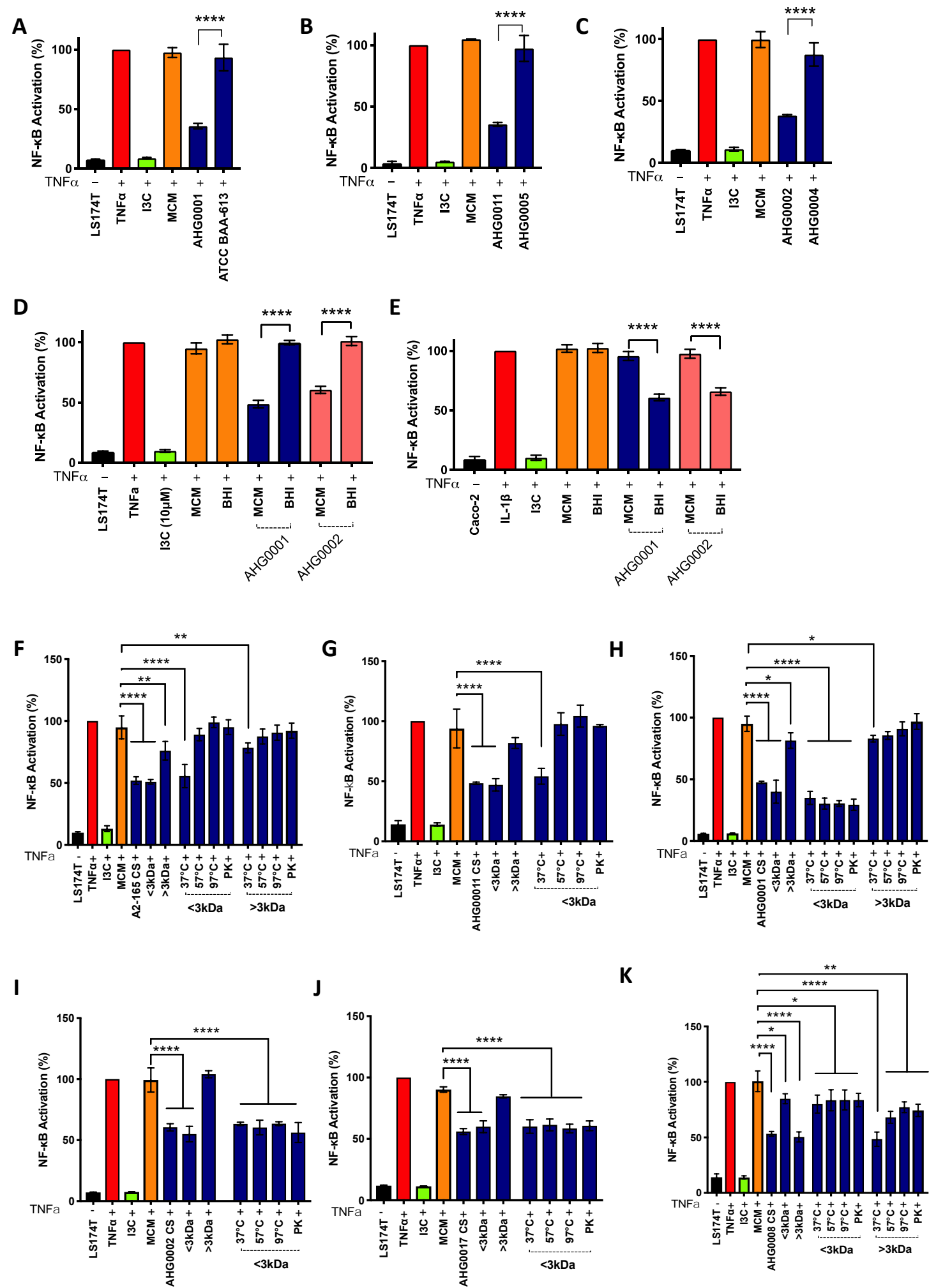

Figure 2A-C. Characterisation of intraspecies variation in $\mathrm{NF}-\kappa \mathrm{B}$ suppressive capacity. The ability of C. bolteae AHG0001 and ATCC BAA-613 (Panel A), C. citroniae AHG0002 and 
561 AHG0004 (Panel B) and C. aldenense AHG0011 and AHG0005 (Panel C) to suppress NF-кB

562 was analysed using the LS174T reporter cells. NF-kB activation was assessed $4 \mathrm{~h}$ after TNF $\alpha$

563 stimulation and the extent of suppression was assessed against sterile medium (mean (SD)).

564 D-E. Characterisation of the effect of growth medium on the NF-kB suppressive capacity of

565 C. bolteae AHG0001 and C. citroniae AHG0002 in LS174T (Panel D) and Caco-2 (Panel E)

566 reporter cell lines. NF- $\mathrm{KB}$ activation was assessed $4 \mathrm{~h}$ after cytokine stimulation and the extent

567 of suppression was assessed against sterile medium. F-K. Characterisation of the bioactive

568 factors produced by F. prausnitzii A2-165 (Panel F), C. aldenense AHG0011 (Panel G), C.

569 bolteae AHG0001 (Panel H), C. citroniae AHG0002 (Panel I), E. limosum AHG0017 (Panel

$570 \mathrm{~J})$ and Pseudoflavonifractor sp. AHG0008 (Panel K). The cell free CS were untreated or

571 subjected to size fractionation, heat and/or proteinase $\mathrm{K}$ treatments as appropriate. NF- $\mathrm{kB}$

572 activation was assessed $4 \mathrm{~h}$ after $\mathrm{TNF} \alpha$ stimulation and the extent of suppression was assessed

573 against sterile medium (mean (SD)). * $p<0.05$, ** $p<0.01$, *** $p<0.001$, **** $p<0.0001$ as

574 determined by one-way ANOVA with Dunnett's multiple comparison test. 
A

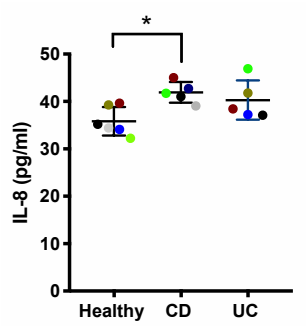

D

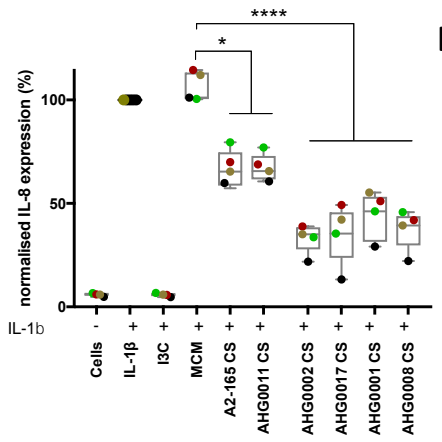

G

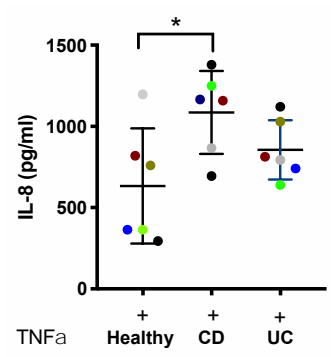

J

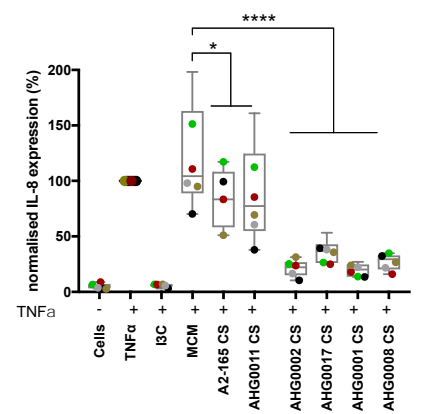

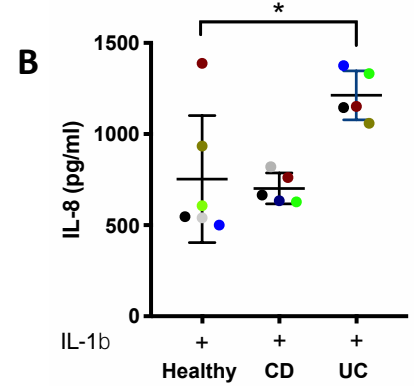

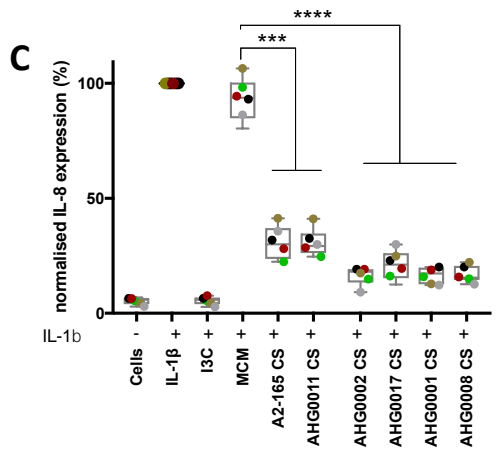

E

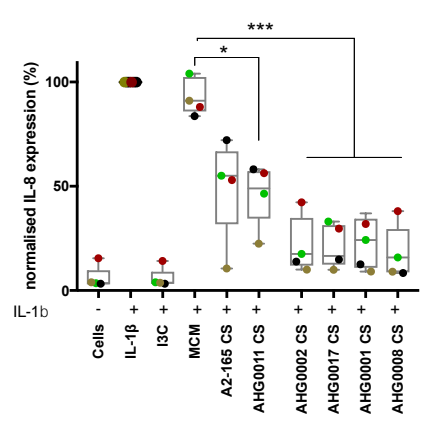

$\mathbf{F}$

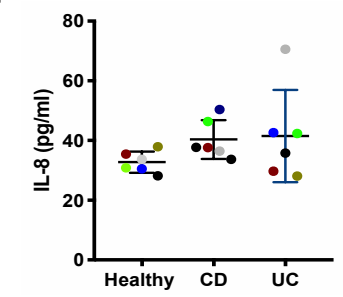

I
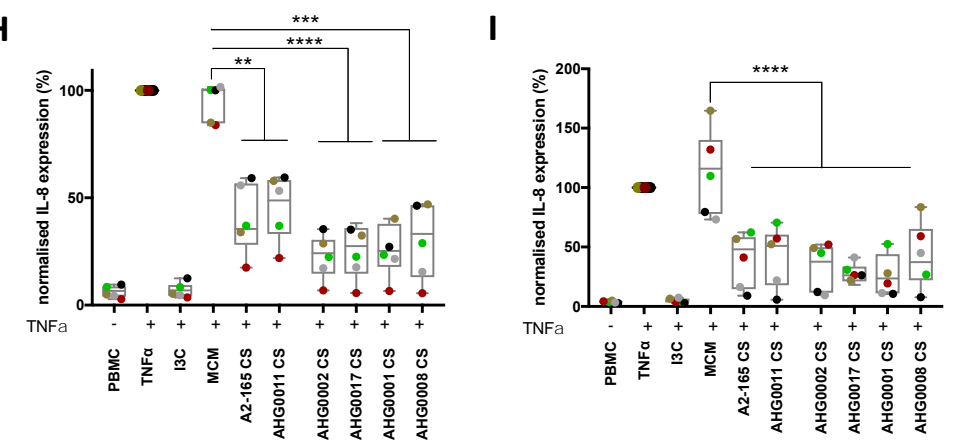

575

576 Figure 3A-B. Analysis of basal IL-8 secretion by healthy, CD and UC derived gut epithelial

577 organoids (Panel A) and following IL-1 $\beta$ stimulation (Panel B). IL-8 secretion was assessed

$57824 \mathrm{~h}$ after cytokine stimulation (mean (SD)). C-E. Analysis of the ability of $F$. prausnitzii A2-

579 165, C. aldenense AHG0011, C. citroniae AHG0002, E. limosum AHG0017, C. bolteae

580 AHG0001 and Pseudoflavonifractor sp. AHG0008 to suppress IL-8 secretion in healthy (Panel 
581 C), CD (Panel D) and UC (Panel E) subject derived gut epithelial organoids. IL-8 secretion

582 was assessed $24 \mathrm{~h}$ after cytokine stimulation and compared against the sterile medium (mean

583 (SD)). F-G. Analysis of basal IL-8 secretion by healthy, CD and UC derived PBMCs (Panel

584 F) and following TNF $\alpha$ stimulation (Panel G). H-J. Analysis of the ability of $F$. prausnitzii

585 A2-165, C. aldenense AHG0011, C. citroniae AHG0002, E. limosum AHG0017, C. bolteae

586 AHG0001 and Pseudoflavonifractor sp. AHG0008 to suppress IL-8 secretion in healthy (Panel

587 H), CD (Panel I) and UC (Panel J) subject derived PBMCs. IL-8 secretion was assessed $24 \mathrm{~h}$

588 after cytokine stimulation and compared against the sterile medium (mean (SD)). $* p<0.05$, **

$589 p<0.01, * * * p<0.001, * * * * p<0.0001$ as determined by one-way ANOVA with Dunnett's

590 multiple comparison test. 


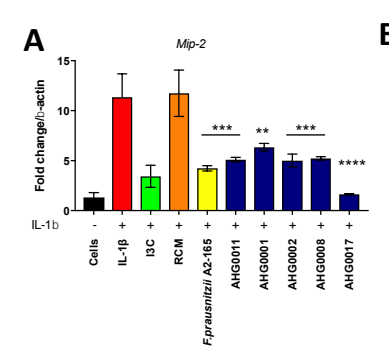

$$
\mathrm{E}
$$
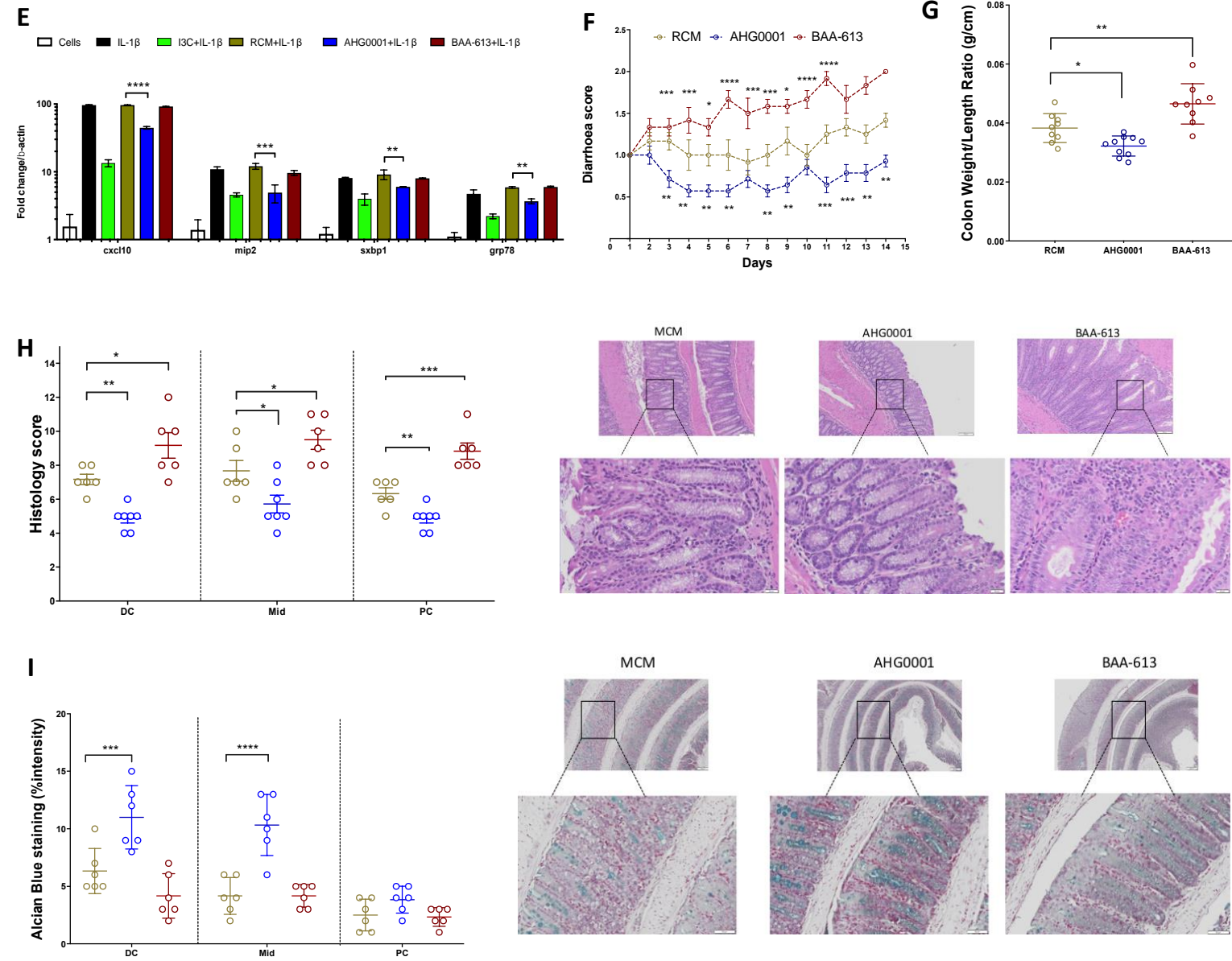

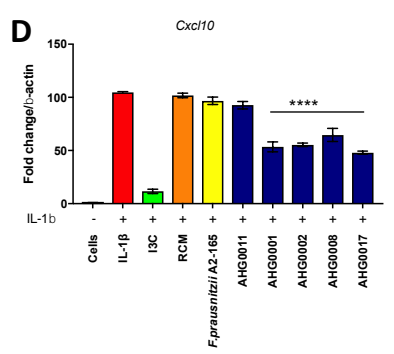

G

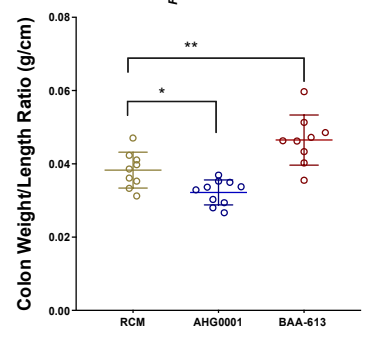

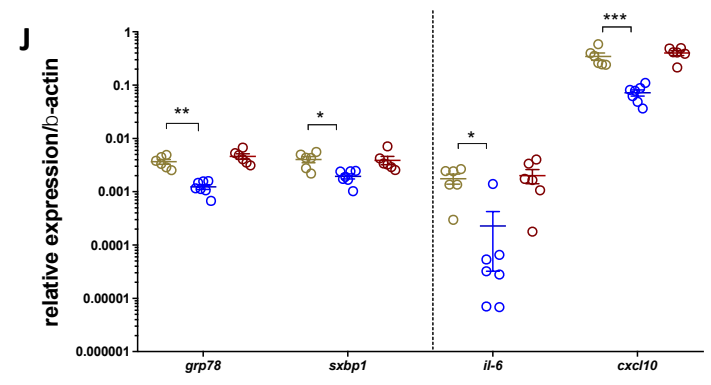

592 Figure 4A-D. Effects of bioactives on pro-inflammatory gene expression using murine derived

593 organoids from C57/BL6 (Panels A-B) and Winnie (Panels C-D) mice. Murine derived

594 organoids were treated with CS for 30mins and then stimulated as appropriate with mIL-1 $\beta$ for 
5956 hours. E. Winnie organoid based qRT-PCR quantification of cxcl10, mip-2, sxbpl and grp78

596 expression following treatment with C. bolteae AHG0001 or C. bolteae BAA-613. F. Effect

597 of daily administration of MCM, C. bolteae AHG0001 CS or C. bolteae BAA-613 CS on

598 diarrhoea score. G. Changes in colon weight/length ratio following treatment with MCM, $C$.

599 bolteae AHG0001 CS or C. bolteae BAA-613 CS. H. Blinded histology scores following

600 treatment with MCM, C. bolteae AHG0001 CS or C. bolteae BAA-613 CS. I. Alcian blue

601 quantification of mucin production in Winnie derived colon sections with representative images

$\mathbf{6 0 2}$ from distal colon. J. Relative gene expression of ER stress markers ( $g r p 78$ and sxbpl) and

603 pro-inflammatory (il-6, cxcl10) genes in colonic tissue sections as analysed by qRT-PCR. ns

604 not significant, * $p<0.05$, ** $p<0.01$, *** $p<0.001$, **** $p<0.0001$. The significance for

605 diarrhoea was determined by comparison with MCM using one-way ANOVA with Dunnett's

606 multiple comparison test. Sidak's multiple comparison tests were used for Figures E-J. 
A

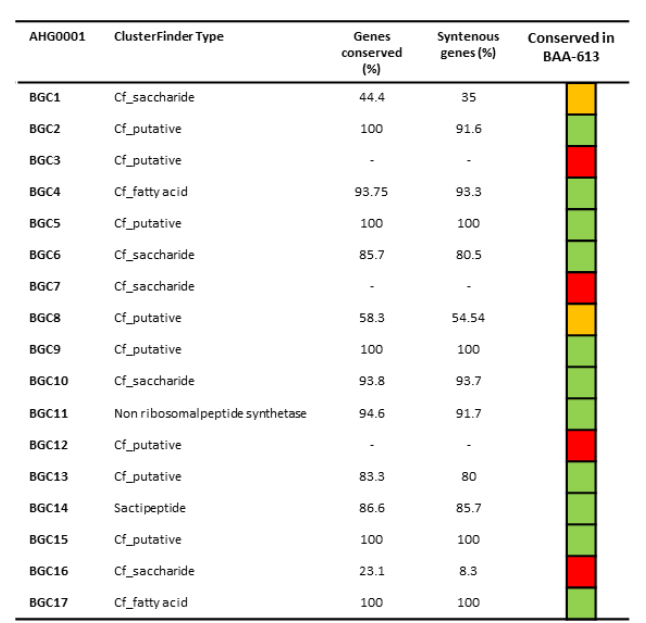

B

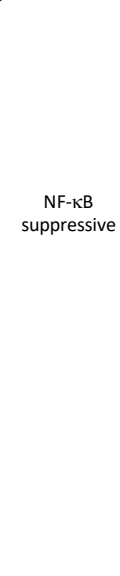

NF-KB
suppressive

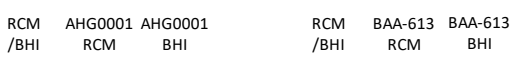

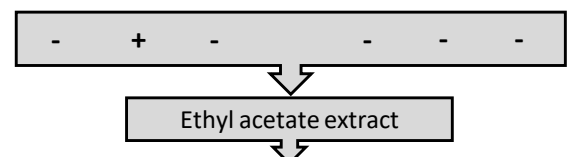

25

GNPS analysis

$\checkmark$

Metabolome maps and identification of unique metabolites

C

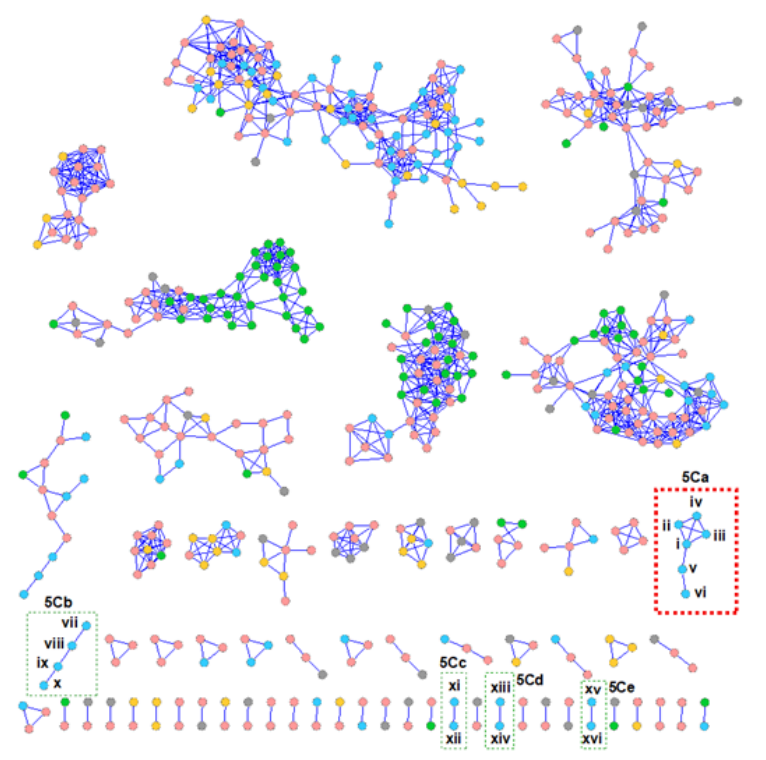

608 Figure 5A. Determination of the extent of $C$. bolteae AHG0001 BGC conservation in $C$.

609 bolteae ATCC BAA-613. The extent of protein (Genes conserved) and syntenic gene pair

610 (Syntenous pairs) conservation was assessed. C. bolteae AHG0001 BGC were classed as being

611 conserved (green), partially conserved (orange) or not conserved (red). B. An overview of the

612 experimental approach used to identify bioactives associated with the NF $\kappa \mathrm{B}$ suppressive

613 activity of $C$. bolteae AHG0001. The presence of the bioactive in the various extractions and

614 filtrates was determined using the LS174T reporter cell assay. C. Molecular networking for

615 EtOAc extracts of $C$. bolteae AHG0001 cultured in MCM (blue nodes) and BHI media (green 
616 nodes); yellow and grey nodes represent compounds from MCM and BHI media only

617 respectively; pink nodes represent compounds common to MCM and BHI extracts or MCM

618 and $\mathrm{BHI}$ media. Boxes $5 \mathrm{Ca}, 5 \mathrm{Cb}, 5 \mathrm{Cc}, 5 \mathrm{Cd}$ and $5 \mathrm{Ce}$ highlight structurally related small

619 molecules clusters (i-vi, vii-x, xi-xii, xiii-xiv and xv-xvi respectively), that are unique to the

620 NF-kB suppressive EtOAc extracts of $C$. bolteae AHG0001 cultured in MCM media. Only

621 the molecules within the 5Ca cluster (red dashed box) are present in semi-preparative HPLC

622 fractions that exhibit NF- $\kappa \mathrm{B}$ suppressive activity. 
Table 1.

\begin{tabular}{|c|c|c|c|c|c|c|c|c|}
\hline $\begin{array}{c}\text { Genome } \\
\text { designation }\end{array}$ & $\begin{array}{c}\text { CheckM marker } \\
\text { lineage (Order (GTDB } \\
\text { Branch)) }\end{array}$ & $\begin{array}{c}\text { Number of } \\
\text { contigs }\end{array}$ & $\begin{array}{c}\text { Genome size } \\
\text { (bp) }\end{array}$ & GC (\%) & $\begin{array}{c}\text { CheckM } \\
\text { completeness (\%) }\end{array}$ & $\begin{array}{c}\text { CheckM } \\
\text { contamination }(\%)\end{array}$ & $\begin{array}{l}\text { Number } \\
\text { of BGC }\end{array}$ & $\begin{array}{c}\text { NCBI accession } \\
\text { number }\end{array}$ \\
\hline $\begin{array}{l}\text { C. bolteae } \\
\text { AHG0001 }\end{array}$ & Clostridiales (UID1342) & 96 & $5,985,600$ & 49.4 & 98.76 & 0.56 & 19 & QYRW00000000 \\
\hline $\begin{array}{l}\text { C. citroniae } \\
\text { AHG0002 }\end{array}$ & Clostridiales (UID1226) & 149 & $6,630,634$ & 48.8 & 99.37 & 0 & 25 & QYRX00000000 \\
\hline $\begin{array}{l}\text { C. aldenense } \\
\text { AHG0011 }\end{array}$ & Clostridiales (UID1226) & 263 & $6,734,822$ & 49.5 & 99.37 & 0 & 24 & QYRY00000000 \\
\hline $\begin{array}{l}\text { E. limosum } \\
\text { AHG0017 }\end{array}$ & Clostridiales (UID1120) & 86 & $4,704,612$ & 47.2 & 99.3 & 0.7 & 31 & QYRZ00000000 \\
\hline
\end{tabular}




\section{Supplementary Results}

Bioactive identification. Based on the strain and medium effects on C. bolteae $\mathrm{NF}-\kappa \mathrm{B}$ suppressive activity we applied a comparative metabolomics approach to identify the bioactive. During purification we noted that direct filtering of CS through a $0.42 \mu \mathrm{m}$ nylon filter resulted in a loss of NF- $\kappa$ B suppressive activity, suggestive of either low water solubility and/or a nonpolar bioactive(s). By contrast, an ethyl acetate (EtOAc) extract derived from the same CS was readily filtered, with the filtrate retaining NF- $\mathrm{B}$ suppressive activity. As a next step in the chemical characterisation, EtOAc extracts were prepared from $C$. bolteae AHG0001 and $C$. bolteae BAA-613 following growth in $\mathrm{MCM}$ and $\mathrm{BHI}$ along with EtOAc extracts from both un-inoculated MCM and BHI media. Each extract was individually subjected to ultra-highperformance liquid chromatography quadrupole time-of-flight mass spectrometric analysis with MS/MS monitoring (UPLC-QTOF-MS/MS), followed by global natural products social molecular networking (GNPS) analysis (Figure 5B), to generate a metabolome map with media controls. These analyses revealed multiple clusters of metabolites that appeared unique to the NF-кB suppressive MCM CS extract however only a single cluster of 6 novel and previously unreported metabolites (i-vi) was not co-clustered with compounds present in culture media, or the non-suppressive BHI CS extract. To confirm whether metabolites i-vi were the target bioactives, a portion of the NF- $\mathrm{BB}$ suppressive EtOAc extract was subjected to fractionation through a reversed-phase analytical HPLC column, with timed collection of 14 fractions. Significantly, NF- $\mathrm{B}$ suppressive activity was localised in the non-polar fractions \#13 and \#14, which UPLC-QTOF analysis using single ion extraction (SIE) monitoring confirmed to colocalise with metabolites i-vi. By contrast, UPLC-QTOF-SIE analysis of other compounds present in media-associated clusters (Figure 5B), revealed they did not uniquely co-localise into the active fractions. 


\section{Supplementary Methods \& Materials}

Bacterial strains, culture conditions and analyses. Anaerobic Firmicutes affiliated bacteria were cultured in anoxic MCM (Lab-Lemco 10 g.L ${ }^{-1}$, Peptone P 10 g.L $\mathrm{L}^{-1}$, Yeast extract 3 g.L $\mathrm{L}^{-1}$, Glucose 5 g.L. ${ }^{-1}$, Starch 2 g.L ${ }^{-1}$, Sodium chloride 5 g.L ${ }^{-1}$, Sodium bicarbonate 15 g.L , $^{-1}$, Resazurin $1 \mathrm{mg} . \mathrm{L}^{-1}$, Cysteine-HCl 1 g. $\mathrm{L}^{-1}$ ) or BHI supplemented with salt solutions 2 and $3^{1}$. F. prausnitzii A2-165 was grown as previously described ${ }^{2}$. A Coy vinyl anaerobic chamber with an anoxic atmosphere $\left(85 \% \mathrm{~N}_{2}: 10 \% \mathrm{CO}_{2}: 5 \% \mathrm{H}_{2}\right)$ was used to process the anaerobic Firmicutes cultures. Bacterial cultures were incubated at $37^{\circ} \mathrm{C}$ for up to 48 hours. Bacterial growth was measured by spectrophotometry $\left(\mathrm{OD}_{600 \mathrm{~nm}}\right)$ using a SPECTRONIC 20D+ Spectrophotometer (ThermoFisher, Sydney).

Bacterial comparative analyses. Phylogenetic trees were constructed by aligning the $16 \mathrm{~S}$ rRNA gene sequences using the SILVA database ${ }^{3}$ and the alignment was then imported into MEGAX $^{4}$. The alignment was refined, and a maximum-likelihood phylogenetic tree constructed displaying the isolate and select reference sequences. The stability of the maximum-likelihood tree was evaluated by 1000 bootstrap replications and Kimura 2parameter modelling. Where necessary, select isolates were subject to whole cell protein profiling to determine intraspecies variations ${ }^{5,6}$. High molecular weight DNA was prepared as previously described ${ }^{7}$. The SPAdes assembler v 3.11.0 was used to quality check, filter and then de novo assemble the sequence data $^{8}$. $\mathrm{CheckM}^{9}$ was used to evaluate the genome sequencing quality by estimating the completeness and contamination based on the phylogenetic assignment of a broad set of marker genes. The C. bolteae AHG0001, $C$. citroniae AHG0002, C. aldenense AHG0011 and E. limosum AHG0017 contigs were ordered using Mauve ${ }^{10}$ with the $C$. bolteae ATCC BAA-613, C. citroniae WAL-17108, Clostridiales bacterium 1_7_47FAA and E. limosum ATCC 8486 genome sequences respectively as 
references. Genome based phylogeny was determined using GTDB $^{11}$ as previously described ${ }^{2}$. Candidate BGC were identified using the antiSMASH webserver ${ }^{12}$ with the ClusterFinder Detection Strictness settings set to "loose" and the Extra Features turned on. Similar candidate BGC were identified in select genomes or the Genbank Database using MultiGeneBlast ${ }^{13}$ in homology search mode. BGCs were considered highly conserved if (i) $\geq 80 \%$ of the genes in an $C$. bolteae AHG0001 BGC were conserved in C. bolteae ATCC BAA-613, with genes defined as being conserved if the query exhibited $\geq 80 \%$ sequence identity over $\geq 80 \%$ of the query length, and; (ii) $\geq 70 \%$ of the potential syntenic genes in a C. bolteae AHG0001 BGC were conserved in a $C$. bolteae ATCC BAA-613 BGC (calculated as ((MultiGeneBlast Total score - No. of Blast hits)/0.5)/(No. of syntenic genes in C. bolteae AHG0001 BGC)). BGC were considered partially conserved if $\geq 40 \%$ of both the genes and potential syntenic genes were conserved.

Measurement of immunomodulatory activities. The LS174T-NF-kBluc or Caco-2-NF-kBluc reporter cell lines were adapted for high-throughput screening using the criterion defined by Zhang et al., ${ }^{14}$ where a Z-factor $\geq 0.5$ represents an excellent assay, thereby providing a sensitive and specific approach to assess the NF- $\kappa \mathrm{B}$ suppressive capacity of the isolates. The Z-factor for each assay was determined and only assays achieving a Z-factor $\geq 0.5$ were processed for further analysis. The high-throughput assays were performed in 96-well microtiter plates as previously described ${ }^{2}$ except that the LS174T reporter cells were stimulated with $50 \mathrm{ng} \cdot \mathrm{ml}^{-1} \mathrm{TNF} \alpha$ and the Caco-2 cell lines were treated with $7.5 \% \mathrm{v} / \mathrm{v}$ CS in complete DMEM medium. NF- $\mathrm{BB}$ driven luciferase expression was assessed using the Pierce ${ }^{\mathrm{TM}}$ Firefly Luc One-Step Glow Assay Kit (ThermoFisher Scientific) according to the manufacturer's instructions. The NF- $\mathrm{BB}$ suppressive isolates were scored and ranked on their Z-score ${ }^{15,16}$.

Organoid culturing and immunomodulatory assays. The colonic biopsies were processed 
and cultured as previously described ${ }^{17}$. Briefly, the biopsies were washed with PBS and digested with collagenase type I $\left(2 \mathrm{mg} \cdot \mathrm{ml}^{-1}\right)$ supplemented with gentamicin $\left(50 \mu \mathrm{g} \cdot \mathrm{ml}^{-1}\right)$ for 15-20 minutes at $37^{\circ} \mathrm{C}$. The isolated crypts were washed with DMEM/F12 medium and centrifuged at $50 \mathrm{x} \mathrm{g}$ for $5 \mathrm{mins}$ at $4^{\circ} \mathrm{C}$. The pellets were then suspended in Basement Membrane Extract (BME, Invitrogen) in a 1:1 ratio. Then, 20 $\mu 1$ of the mixture was plated in a 24 well tissue culture plate and cultured in 50\% L-WRN conditioned medium. The crypts were expanded by serial culture until sufficient numbers were obtained for experimentation. To assess the ability of the CS to suppress IL- 8 secretion the organoids were seeded in a 48 well plate and grown for 48 hours. Then, organoids were treated with $10 \% \mathrm{v} / \mathrm{v}$ of select CS in $50 \%$ L-WRN conditioned medium for 30 min and subsequently stimulated with rhIL-1 $\beta$ (50 ng.ml ${ }^{1}$ ) for 24 hours before quantifying IL-8 in the supernatant. Cytotoxicity was assessed using the CytoTox 96® Non-Radioactive Cytotoxicity Assay.

Colonic tissues from C57BL/6 and Winnie mice $(n=2)$ were segmented and the crypts were isolated and cultured. Briefly, the tissues were segmented and washed with PBS, followed by EDTA $(8 \mathrm{mM})$ digestion for 1 hour at $4{ }^{\circ} \mathrm{C}$ and further digested with collagenase type $\mathrm{I}\left(2 \mathrm{mg} \cdot \mathrm{ml}^{-}\right.$

$\left.{ }^{1}\right)$ (Thermo Fisher Scientific) supplemented with gentamicin $\left(50 \mu \mathrm{g} \cdot \mathrm{ml}^{-1}\right)$ for $15-20$ minutes at $37^{\circ} \mathrm{C}$. The isolated crypts were washed with complete F12 medium (Identical to complete media except DMEM/F12 was used instead of DMEM) and centrifuged at $50 \mathrm{x}$ g for 5 mins at $4^{\circ} \mathrm{C}$. The pellets were then suspended in BME in a 1:1 ratio. Then, $20 \mu 1$ of the mixture was plated in a 24 well tissue culture plate and cultured in 50\% L-WRN conditioned medium. The crypts were expanded by serial culture until sufficient numbers were obtained for experimentation.

Peripheral Blood Mononuclear Cell (PBMC) isolation and immunomodulatory assays. PBMCs were isolated by Ficoll gradient density centrifugation. Briefly, $20 \mathrm{ml}$ of freshly drawn 
blood was diluted in phosphate buffered saline (1:2) and well mixed. The diluted blood was then carefully layered over Ficoll paque. The tubes were centrifuged without brakes at $400 \mathrm{~g}$ for 20 minutes at $20^{\circ} \mathrm{C}$. The interphase containing mononuclear cells were transferred into a new tube and washed twice in PBS. Prepared cells were stored in liquid nitrogen until required.

Animal experiments. Disease activity was assessed using established protocols. Briefly, the body weights of the mice as well as diarrhoea and rectal bleeding were monitored and recorded daily. Diarrhoea scoring was interpreted as follows: $0=$ no diarrhoea, solid stool; $0.5=$ very mild diarrhoea, moist but formed stool; 1 = mild diarrhoea, formed but easily bisected by pressure applied with pipette tips; $1.5=$ diarrhoea, no fully formed stools, and; $2=$ severe, watery diarrhoea with minimal solid present. For histology scoring, the whole colon was rolled, fixed in $10 \%$ neutral buffered formalin, and paraffin embedded and sectioned and stained with Haematoxylin and Eosin (H\&E) and Alcian blue. Blind assessment of histologic inflammation (increased leukocyte infiltration, neutrophil counts, depletion of goblet cells, crypt abscesses, aberrant crypt architecture, increased crypt length, and epithelial cell damage and ulceration) for proximal, mid and distal colon was performed as previously described. To quantify in vivo gene expression, the distal colon was snap frozen and homogenised in TRIzol. RNA was extracted using the Bioline RNA extraction kit according to manufacturer's instructions. RNA concentration was measured using a Nanodrop 1000 spectrophotometer, followed by cDNA synthesis using $1 \mu \mathrm{g}$ of RNA and the iScript cDNA synthesis kit (BioRad). The expression of genes of interest (Supplementary Table 2) were analysed using quantitative real time PCR (qRT-PCR) as previously described ${ }^{24,25} \cdot \mathrm{C}_{\mathrm{t}}$ values were generated, and relative quantitation was determined by the $\Delta \mathrm{C}_{\mathrm{t}}$ method.

GNPS Analyses. UHPLC conditions involved $0.5 \mathrm{~mL} \cdot \mathrm{min}^{-1}$ gradient elution from $10 \%$ $\mathrm{CH}_{3} \mathrm{CN} / \mathrm{H}_{2} \mathrm{O}$ to $100 \% \mathrm{CH}_{3} \mathrm{CN}$ over a period of $4.5 \mathrm{~min}$, with constant $0.1 \%$ formic acid, 
through an Agilent SB-C $81.7 \mu \mathrm{m}, 2.1 \times 150 \mathrm{~mm}$ column (Agilent Technologies Inc., Mulgrave, VIC, Australia). The source parameters were: electrospray positive ionisation; mass range of m/z 50-1700; scan rate $10 \times$ per sec; MS/MS scan rate $3 \times$ per sec; fixed collision energy 40 $\mathrm{eV}$; source gas temperature $325^{\circ} \mathrm{C}$; gas flow $10 \mathrm{~L} \cdot \mathrm{min}^{-1}$; and nebulizer $20 \mathrm{psig}$. The scan source parameters were: VCap 4000; fragmentor 100; skimmer 45; and octopole RF Peak 750.

Analytical fractionation of $N \boldsymbol{N}-\boldsymbol{\kappa} B$ suppressive extract. An EtOAc extract (3 mg) of $C$. bolteae AHG0001 cultivated on MCM medium was subjected to analytical HPLC (Agilent Zorbax SB-C8, $5 \mu \mathrm{m}, 4.6 \mathrm{~mm} \times 150 \mathrm{~mm}$ column, gradient elution at $1 \mathrm{~mL} \cdot \mathrm{min}^{-1}$ from $10 \%$ $\mathrm{MeCN} / \mathrm{H}_{2} \mathrm{O}$ to $100 \% \mathrm{MeCN}$ over 15 min followed by 2 min wash with $100 \% \mathrm{MeCN}$, without TFA modifier) to yield 17 fractions. Only fractions 14-17 demonstrated an ability to suppress $\mathrm{NF}-\kappa \mathrm{B}$ activity. 
A

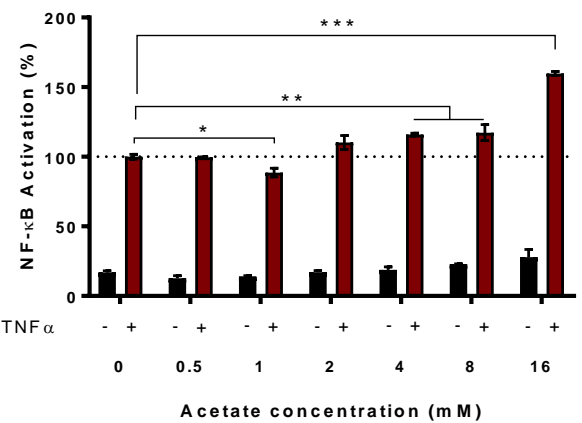

C

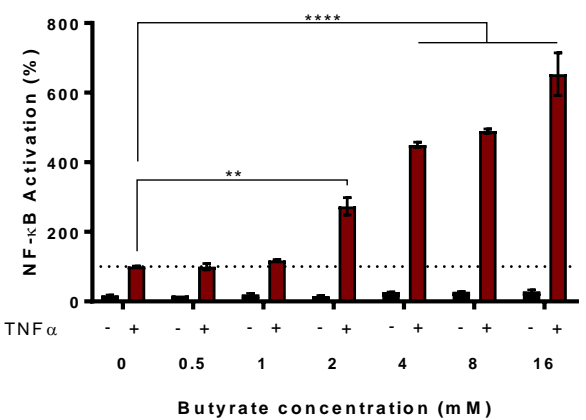

E
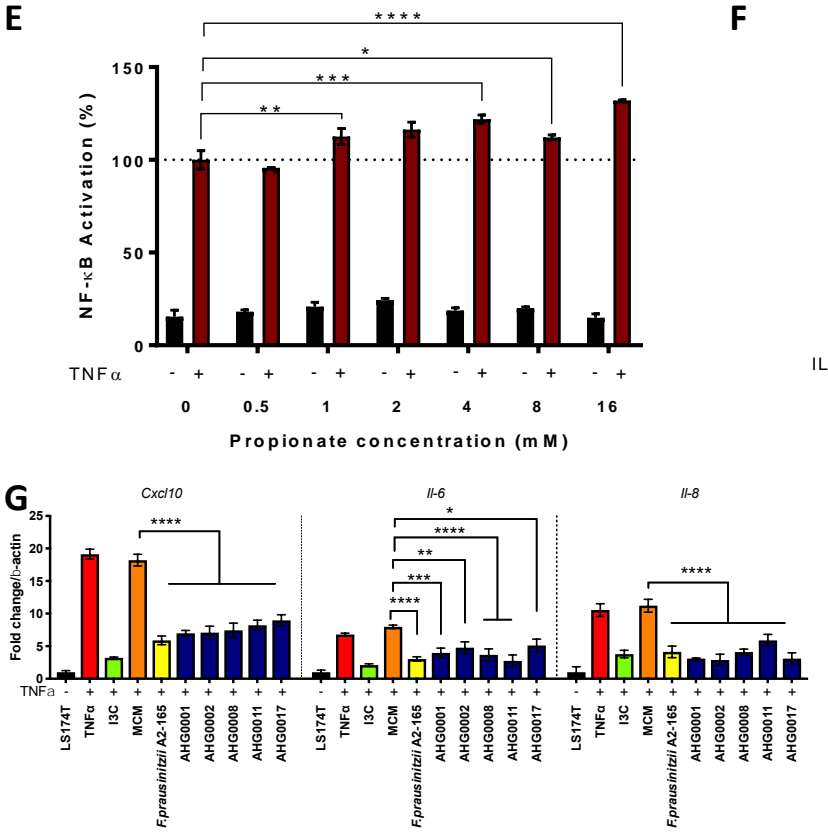

B

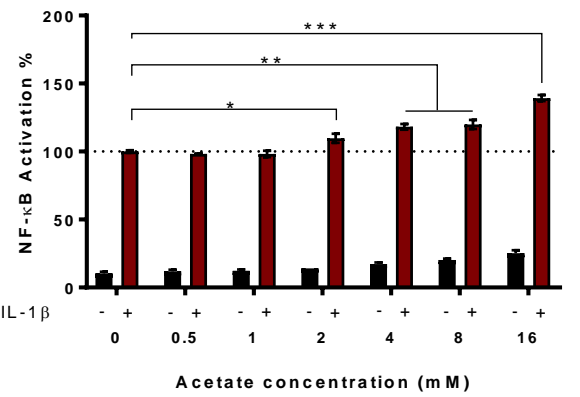

D

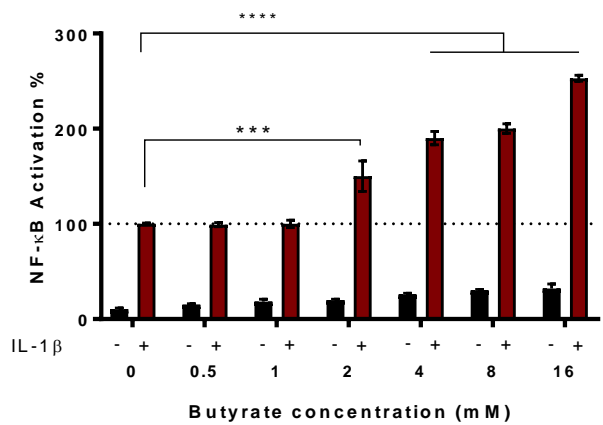

$\mathbf{F}$

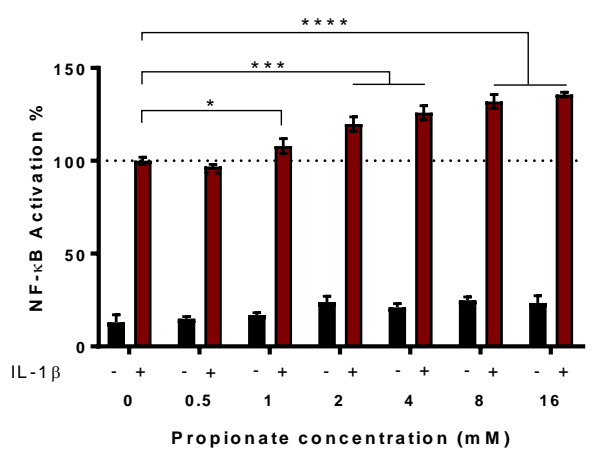

H

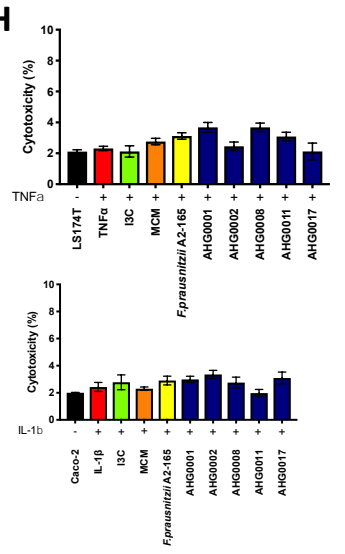

Supplementary Figure 1A-F. Assessment of the ability of acetate (Panels A-B), butyrate (Panels C-D) or propionate (Panels E-F) to suppress NF- $\kappa \mathrm{B}$ activation in unstimulated or cytokine stimulated LS174T and Caco-2 reporter cells. NF-kB activation was assessed $6 \mathrm{~h}$ after 
$\mathrm{TNF} \alpha$ stimulation and the extent of suppression was assessed against sterile medium (mean (SD)). G. LS174T based qRT-PCR confirmatory assay of the hits identified from the first pass screen (mean (SD)). F. prausnitzii A2-165 and the validated hits suppress IL-1 $\beta$ induced cxcl10, il6 and il8 expression in LS174T cells. H-I. Analysis of the cytotoxicity of the CS prepared from the NF- $\kappa \mathrm{B}$ suppressive strains in LS174T (Panel H) and Caco-2 (Panel I) reporter cells. CS prepared from these strains did not exhibit cytotoxic effects (mean (SD)). $p<0.05, * * p<0.01, * * * p<0.001, * * * * p<0.0001$ as determined by one-way ANOVA with Dunnett's multiple comparison test. 
A

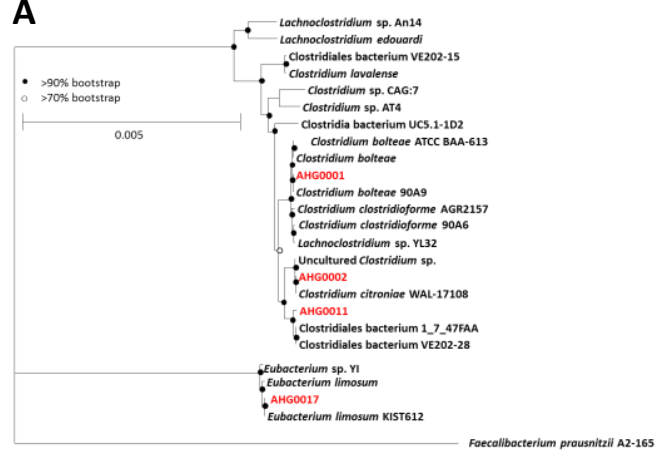

D

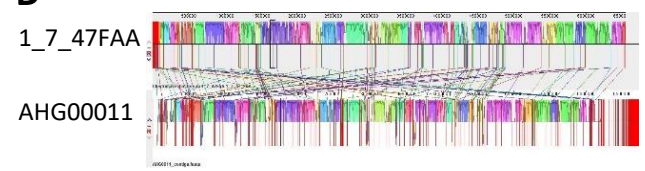

B

BAA-613

AHG0001

C

WAL-17108

AHG0002

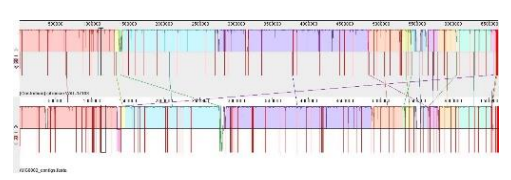

E

ATCC 8486

AHG00017

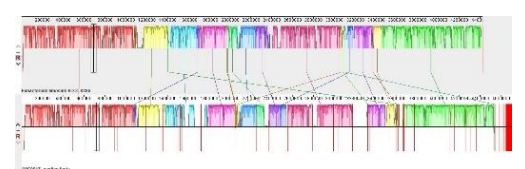

Supplementary Figure 2A. GTDB-based phylogeny of C. bolteae AHG0001, C. citroniae AHG0002, C. aldenense AHG0011 and E. limosum AHG0017 (red typeface) as determined from the concatenation of 120 universal bacterial-specific marker genes. Representative strains are included for comparative purposes (black typeface). The bootstrap values are indicated using a cut-off of $>70$ or $>90 \%$. B-E. The extent of genome synteny between $C$. bolteae ATCC BAA-613 and C. bolteae AHG0001 (Panel B), C. citroniae WAL-17108 and C. citroniae AHG0002 (Panel C), C. aldenense 1_7_47FAA and C. aldenense AHG0011, and; E. limosum ATCC8486 and E. limosum AHG0017. The red lines indicate the boundaries of chromosomes, plasmids or contigs. 
A
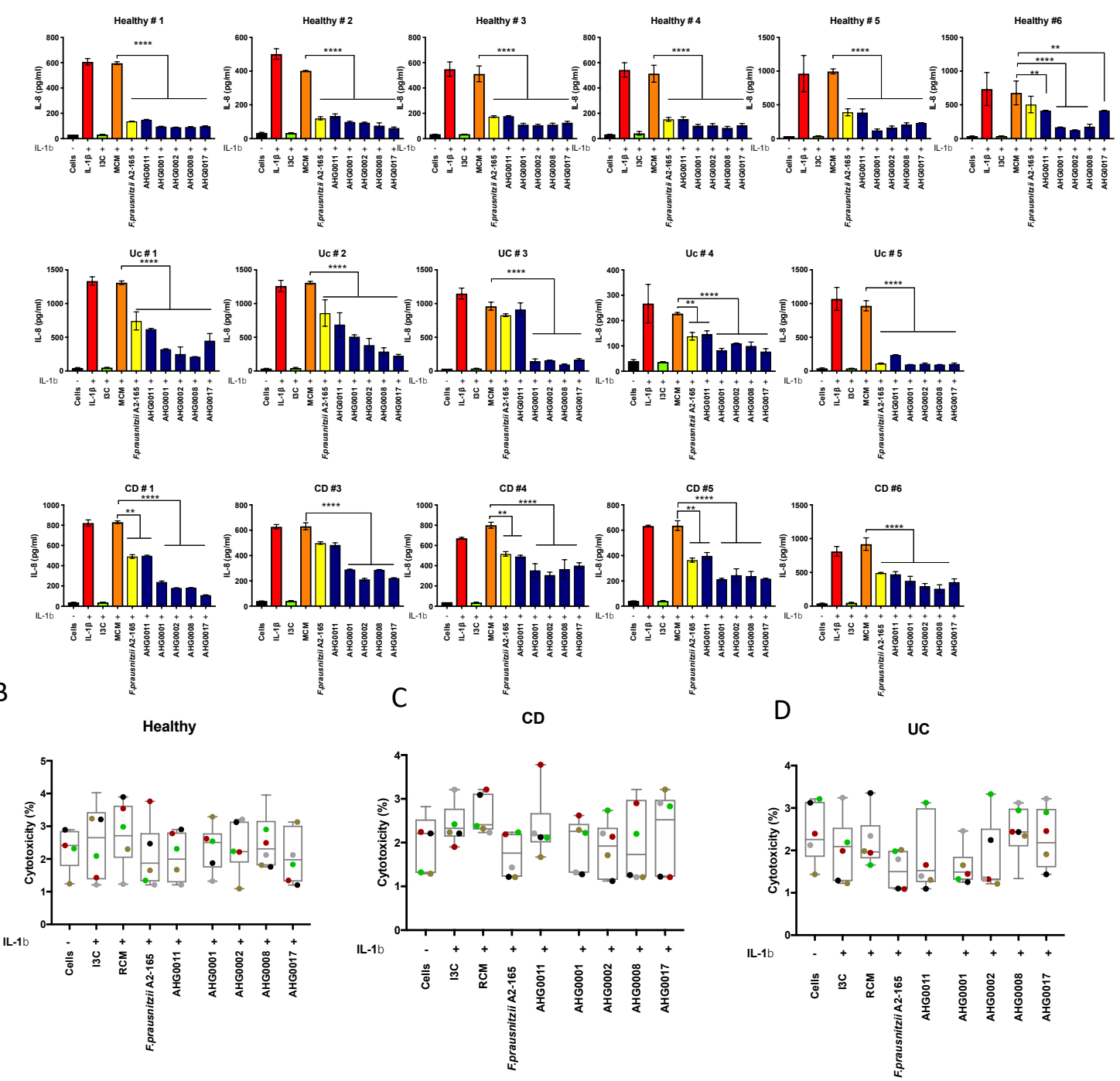

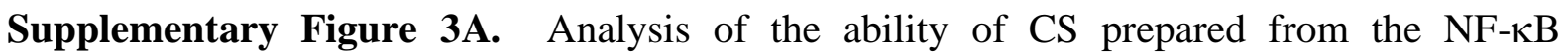
suppressive strains to suppress IL-8 secretion in organoids produced from healthy (Healthy, $\mathrm{n}=6)$, Crohn's disease $(\mathrm{CD}, \mathrm{n}=5)$ or ulcerative colitis $(\mathrm{UC}, \mathrm{n}=5)$ subjects. B. Analysis of the cytotoxicity of the CS prepared from the NF- $\mathrm{kB}$ suppressive strains in organoids produced from healthy (Healthy, $n=6)$, Crohn's disease $(C D, n=5)$ or ulcerative colitis (UC, n=5) subjects. ${ }^{* *} p<0.01, * * * * p<0.0001$ as determined by one-way ANOVA with Dunnett's multiple comparison test. 
A
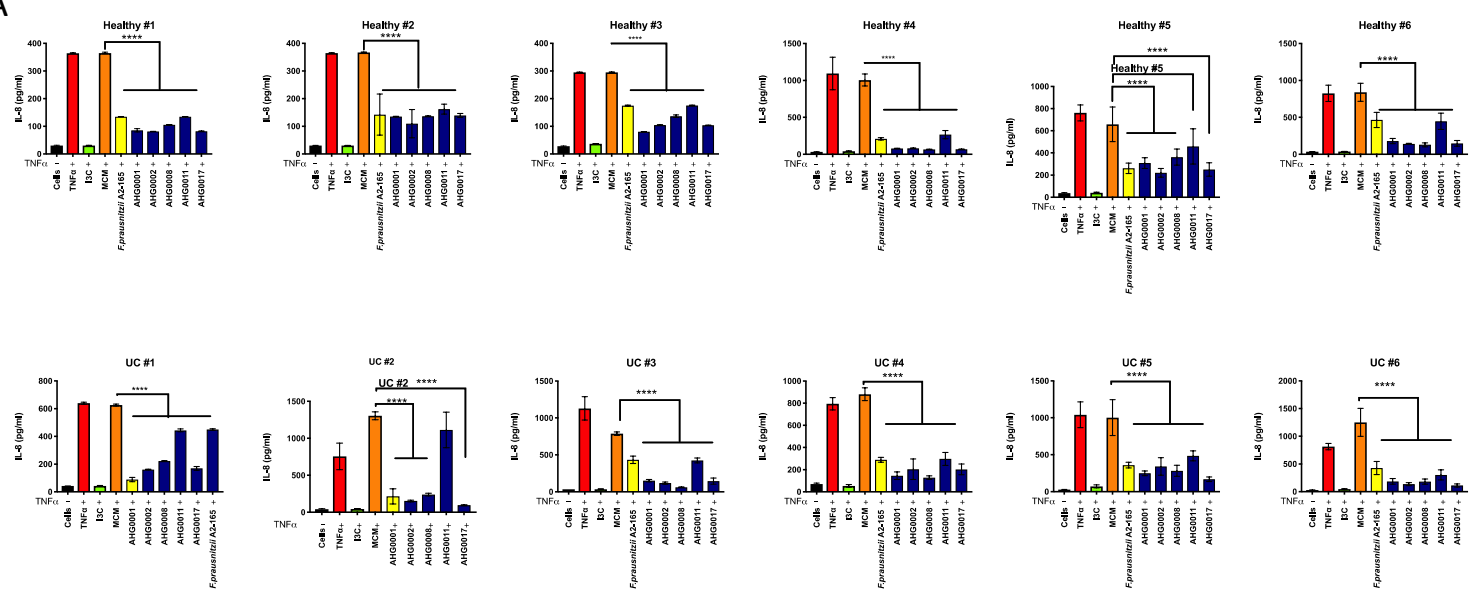

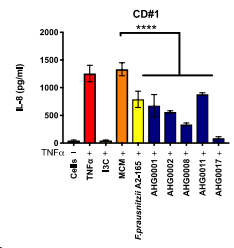

B

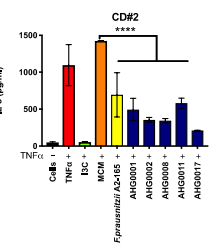

Healthy

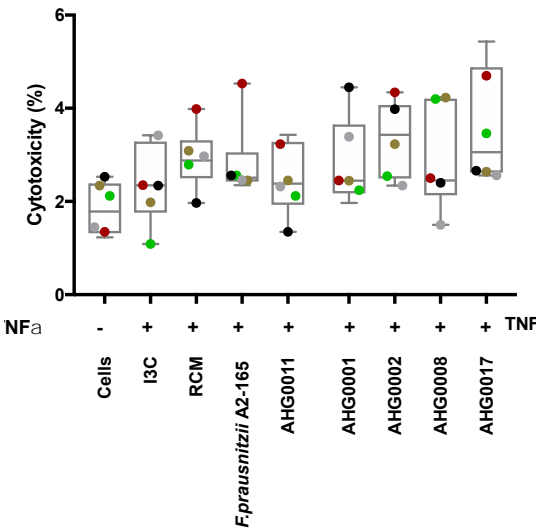

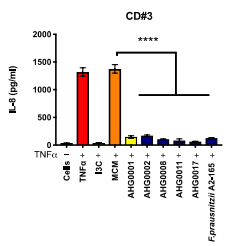

C

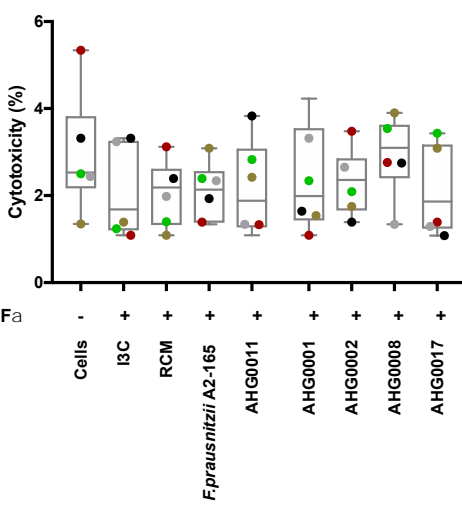

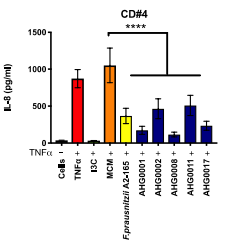

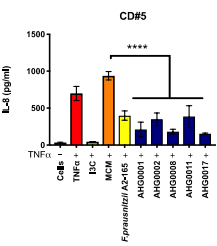

D

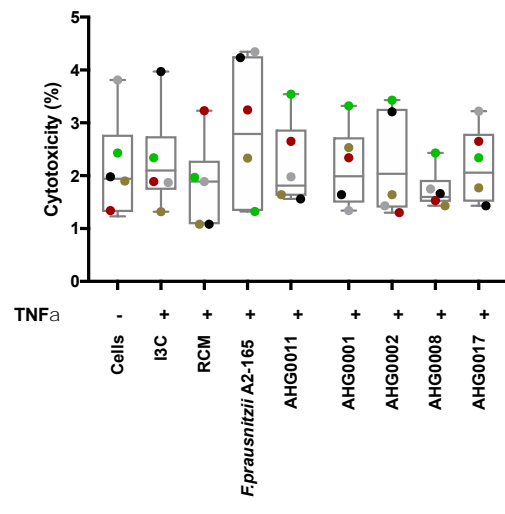

Supplementary Figure 4. Analysis of the ability of CS prepared from the NF- $\kappa B$ suppressive strains to suppress IL-8 secretion in PBMCs prepared from healthy (Healthy, $n=6$ ), Crohn's disease $(C D, n=6)$ or ulcerative colitis $(U C, n=6)$ subjects. B. Analysis of the cytotoxicity of the CS prepared from the NF- $\mathrm{BB}$ suppressive strains in PBMCs produced from healthy (Healthy, $\mathrm{n}=6)$, Crohn's disease $(\mathrm{CD}, \mathrm{n}=5)$ or ulcerative colitis $(\mathrm{UC}, \mathrm{n}=5)$ subjects. $* p<0.05$, **** $p<0.0001$ as determined by one-way ANOVA with Dunnett's multiple comparison test. 

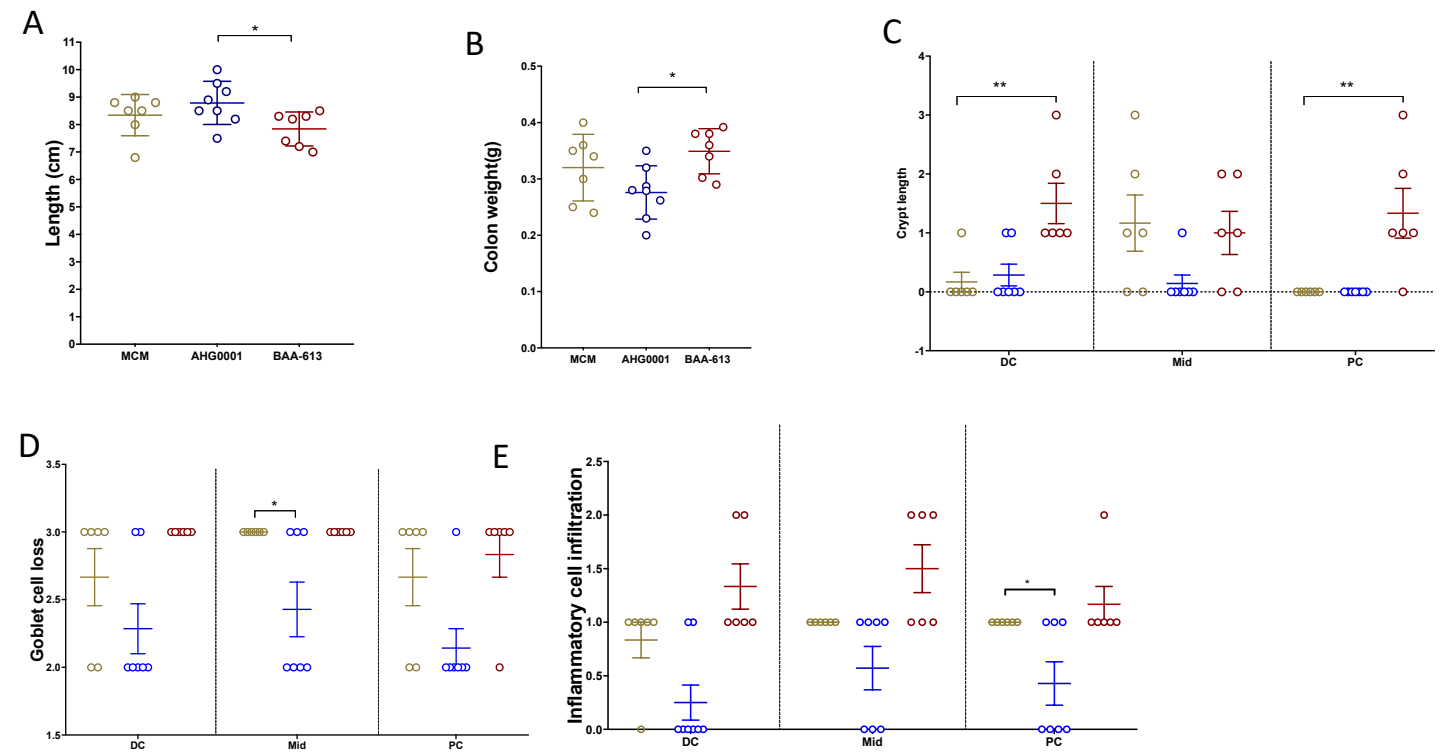

$\mathrm{F}$

\begin{tabular}{|l|}
\hline Region Examined \\
\hline Crypt Architecture \\
$0=$ normal \\
$1=$ irregular \\
$2=$ moderate crypt loss (10-50\%) \\
$3=$ severe crypt loss (50-90\%) \\
$4=$ small/medium sized ulcers (<10 crypt widths) \\
$5=$ large ulcers ( $>10$ crypt widths) \\
\hline Crypt Abscesses \\
$0=$ none \\
$1=1-5$ \\
$2=6-10$ \\
$3=>10$ \\
\hline Crypt Length \\
PC $-0=<150$ uM, $1=150-200$ um, $2=200-250,3=250-300,4=>300$ \\
MC $-0=<250$ uM, $1=250-300$ um, $2=300-400,3=350-400,4=>400$ \\
DC $-0=<200$ uM, $1=200-250$ um, $2=250-300,3=300-350,4=>350$ \\
\hline Tissue Damage \\
$0=$ no damage \\
$1=$ discrete lesions \\
$2=$ mucosal erosions \\
$3=$ extensive mucosal damage \\
\hline Goblet Cell Loss \\
$0=$ normal $<<10 \%$ loss \\
$1=10-25 \%$ \\
$2=25-50 \%$ \\
$3=>50 \%$ \\
\hline Inflammatory Cell Infiltration \\
$0=$ occasional infiltration \\
$1=$ increasing leukocytes in lamina propria \\
$2=$ confluence of leukocytes extending to submucosa \\
$3=$ transmural extension of inflammatory infiltrates \\
\hline Lamina Propria Neutrophils (PMN) \\
$0=0-5$ PMNs $/$ HPF \\
$1=6-10$ \\
$2=11-20$ \\
$3=>20$ \\
\hline
\end{tabular}

Supplementary Figure 5. Histological colonic inflammation sub-scores following the treatment with MCM, C. bolteae AHG0001 and C. bolteae BAA-613. A. Colon length B. Colon weight C. Crypt length D. Goblet cell loss E. Inflammatory cell infiltration F. Criteria for histology sub score $* p<0.05,{ }^{*} p<<0.01$ as determined by one-way ANOVA with Dunnett's multiple comparison test. 


\section{A $B G C 1$}

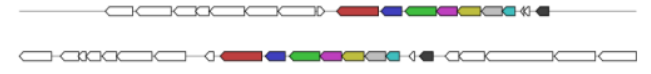

BGC 2

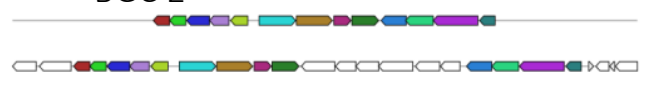

BGC 4

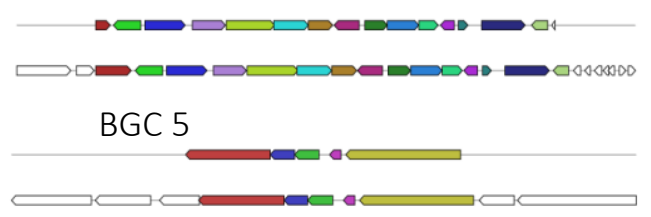

BGC 6

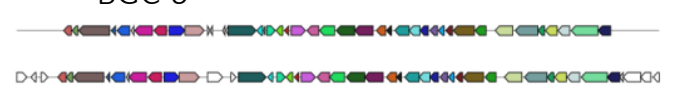

BGC 8

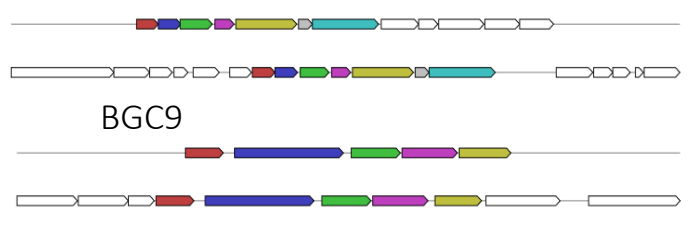

B

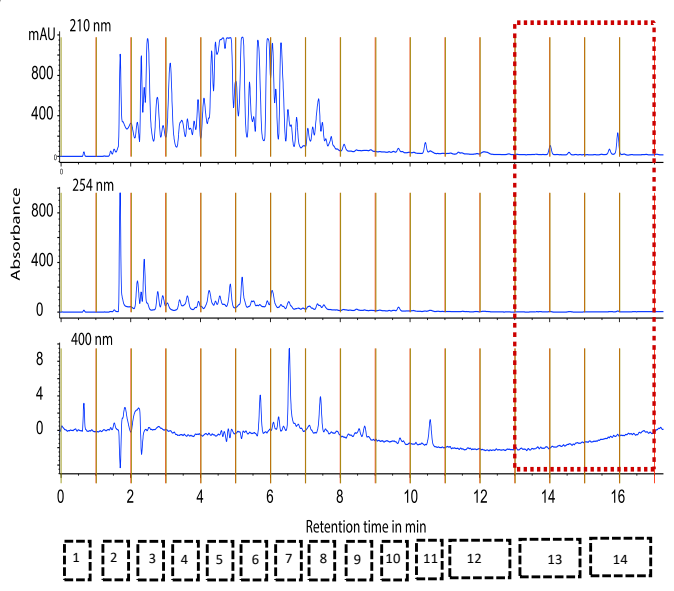

BGC 10

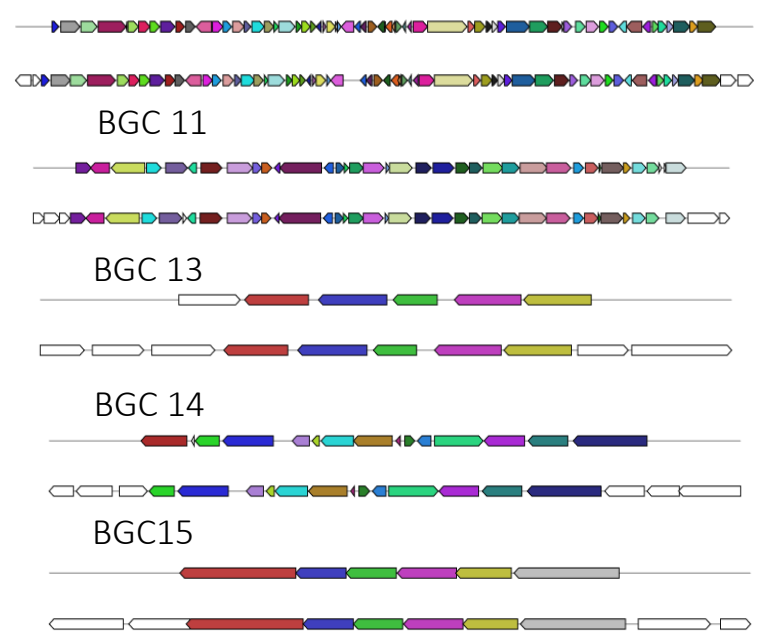

BGC 16

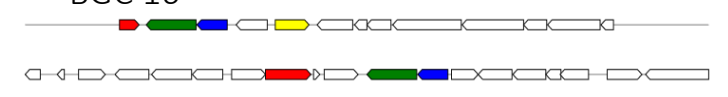

BGC 17

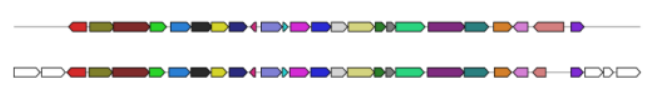

C

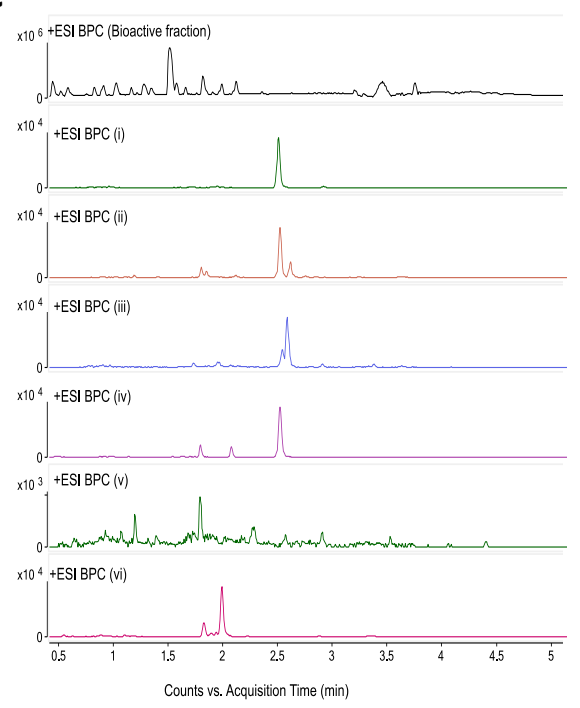

Supplementary Figure 6A. Gene organisation of the highly and partially conserved BGCs between C. bolteae AHG0001 (top) and C. bolteae ATCC BAA-613 (bottom). BGC 12 and BGC 13 are contiguous in C. bolteae AHG0001 and this BGC disrupted by a transposon insertion in $C$. bolteae ATCC BAA-613. B. Semi-preparative HPLC fractionation of the EtOAc extract of $C$. bolteae AHG0001 cultured in MCM media. The collected fractions are indicated with black dashed boxes and the red dashed box represents the NF- $\kappa \mathrm{B}$ suppressive 
fractions. C. UPLC-QTOF single ion extraction chromatograms demonstrating that small molecules i-vi (Figure 5Ca) are present in the combined NF- $\kappa \mathrm{B}$ suppressive semi-preparative HPLC fractions (see Panel B). BPC $=$ selected base peak $(\mathrm{m} / \mathrm{z}$ molecular ion) chromatogram. Small molecules vii-xvi were not present in the active fraction. 


\section{Supplementary Table 1.}

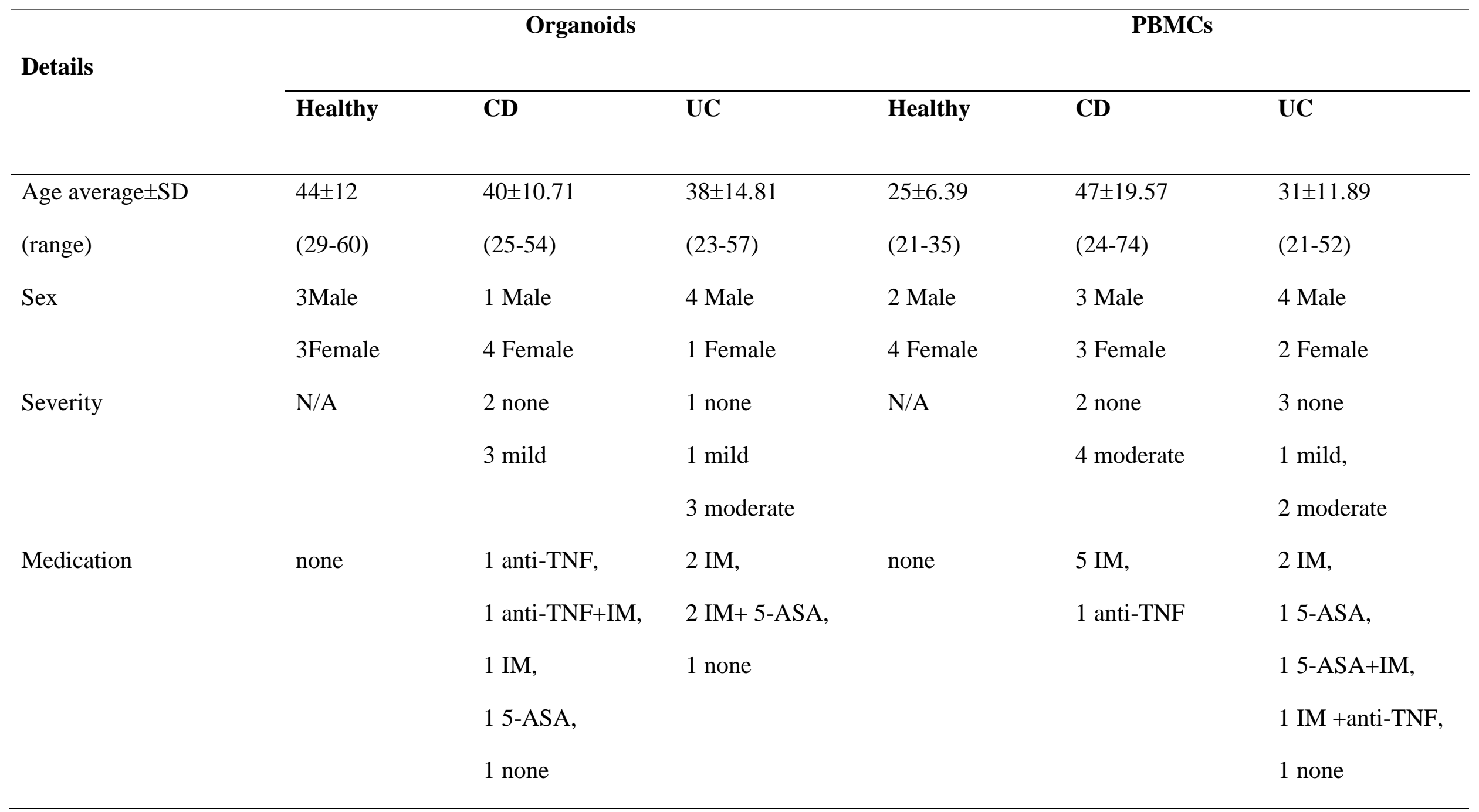

$\mathrm{IM}=$ Immunomodulator, 5-ASA = 5-aminosalicylic acid 


\section{Supplementary Table 2}

\begin{tabular}{|c|c|c|c|}
\hline Primer name & Primer target & Primer sequence $5^{\prime}-3^{\prime}$ & Reference \\
\hline$h$-cxcllo & Human $\operatorname{cxcl} 10$ & $\begin{array}{l}\text { AGC AGA GGA ACC TCC AGT CT } \\
\text { TGT GGT CCA TCC TTG GAA GC }\end{array}$ & 2 \\
\hline h-il-6 & Human il-6 & $\begin{array}{l}\text { CCA CTC ACC TCT TCA GAA CG } \\
\text { CAT CTT TGG AAG GTT CAG GTT G }\end{array}$ & 2 \\
\hline$h-i l-8$ & Human il-8 & $\begin{array}{l}\text { ACT CCA AAC CTT TCC ACC C } \\
\text { CCC TCT TCA AAA ACT TCT CCA C }\end{array}$ & 2 \\
\hline$h$ - $\beta$-actin & Human $\beta$-actin & $\begin{array}{l}\text { CCT GTA CGC CAA CAC AGT GC } \\
\text { ATA CTC CTG CTT GCT GAT CC }\end{array}$ & 18 \\
\hline$m$-cxcllo & Murine $\operatorname{cxcl} 10$ & $\begin{array}{l}\text { TCC TTG TCC TCC CTA GCT CA } \\
\text { ATA ACC CCT TGG GAA GAT GG }\end{array}$ & 19 \\
\hline m-mip2 & Murine mip2 & $\begin{array}{l}\text { ACC ACC AGG CTA CAG GGG CT } \\
\text { GGT CCT GGG GGC GTC ACA CT }\end{array}$ & 19 \\
\hline$m$-sxbpl & Murine sxbpl & $\begin{array}{l}\text { GAG TCC GCA GCA GGT GC } \\
\text { CAA AAG GAT ATC AGA CTC AGA ATC TGA A }\end{array}$ & 19 \\
\hline$m$-grp78 & Murine grp78 & $\begin{array}{l}\text { TGC TGC TAG GCC TGC TCC GA } \\
\text { CGA CCA CCG TGC CCA CAT CC }\end{array}$ & 20 \\
\hline$m$ - $\beta$-actin & Murine $\beta$-actin & $\begin{array}{l}\text { GAA ATC GTG CGT GAC ATC AAA } \\
\text { CAC AGG ATT CCA TAC CCA AGA }\end{array}$ & 21 \\
\hline
\end{tabular}




\section{References}

1. McSweeney, C.S., Denman, S.E. \& Mackie, R.I. in Methods in gut microbial ecology for ruminants. (eds. H.P.S. Makkar \& C.S. McSweeney) 23-37 (Springer, Dordrecht; 2005).

2. Ó Cuív, P. et al. Enterococcus faecalis AHG0090 is a genetically tractable bacterium and produces a secreted peptidic bioactive that suppresses nuclear factor kappa B activation in human gut epithelial cells. Front. Immunol. 9 (2018).

3. Quast, C. et al. The SILVA ribosomal RNA gene database project: improved data processing and web-based tools. Nucleic Acids Res. 41, D590-D596 (2013).

4. Kumar, S., Stecher, G., Li, M., Knyaz, C. \& Tamura, K. MEGA X: Molecular evolutionary genetics analysis across computing platforms. Mol. Biol. Evol. 35, 15471549 (2018).

5. Ismail, Y. et al. Investigation of the enteric pathogenic potential of oral Campylobacter concisus strains isolated from patients with inflammatory bowel disease. PLoS One 7, e38217 (2012).

6. Mahendran, V. et al. The prevalence and polymorphisms of zonula occluden toxin gene in multiple Campylobacter concisus strains isolated from saliva of patients with inflammatory bowel disease and controls. PLoS One 8, e75525 (2013).

7. Ó Cuív, P. et al. The effects from DNA extraction methods on the evaluation of microbial diversity associated with human colonic tissue. Microb. Ecol. 61, 353-362 (2011).

8. Bankevich, A. et al. SPAdes: a new genome assembly algorithm and its applications to single-cell sequencing. J. Comput. Biol. 19, 455-477 (2012).

9. Parks, D.H., Imelfort, M., Skennerton, C.T., Hugenholtz, P. \& Tyson, G.W. CheckM: assessing the quality of microbial genomes recovered from isolates, single cells, and metagenomes. Genome Res. (2015).

10. Darling, A.E., Mau, B. \& Perna, N.T. progressiveMauve: multiple genome alignment with gene gain, loss and rearrangement. PLoS One 5, e11147 (2010).

11. Parks, D.H. et al. A standardized bacterial taxonomy based on genome phylogeny substantially revises the tree of life. Nat. Biotechnol. 36, 996 (2018).

12. Weber, T. et al. antiSMASH 3.0-a comprehensive resource for the genome mining of biosynthetic gene clusters. Nucleic Acids Res. 43, W237-243 (2015).

13. Medema, M.H., Takano, E. \& Breitling, R. Detecting sequence homology at the gene cluster level with MultiGeneBlast. Mol. Biol. Evol. 30, 1218-1223 (2013).

14. Zhang, J.-H., Chung, T.D.Y. \& Oldenburg, K.R. A simple statistical parameter for use in evaluation and validation of high throughput screening assays. Journal of Biomolecular Screening 4, 67-73 (1999).

15. Junker, L.M. \& Clardy, J. High-throughput screens for small-molecule inhibitors of Pseudomonas aeruginosa biofilm development. Antimicrobial agents and chemotherapy 51, 3582-3590 (2007).

16. Malo, N., Hanley, J.A., Cerquozzi, S., Pelletier, J. \& Nadon, R. Statistical practice in high-throughput screening data analysis. Nature biotechnology 24, 167-175 (2006).

17. VanDussen, K.L. et al. Development of an enhanced human gastrointestinal epithelial culture system to facilitate patient-based assays. Gut 64, 911-920 (2015).

18. Maudet, C. et al. Functional high-throughput screening identifies the miR-15 microRNA family as cellular restriction factors for Salmonella infection. Nat Commun 5, 4718 (2014).

19. Hasnain, S.Z. et al. Glycemic control in diabetes is restored by therapeutic manipulation of cytokines that regulate beta cell stress. Nat Med 20, 1417-1426 
(2014).

20. Gulhane, M. et al. High fat diets induce colonic epithelial cell stress and inflammation that is reversed by IL-22. Sci. Rep. 6, 28990 (2016).

21. Heazlewood, C.K. et al. Aberrant mucin assembly in mice causes endoplasmic reticulum stress and spontaneous inflammation resembling ulcerative colitis. PLoS Med. 5, e54 (2008). 